\title{
Spatial heterogeneity and evolution of fecundity-affecting traits
}

\author{
Kalle Parvinen ${ }^{1,2,3}$, Hisashi Ohtsuki ${ }^{3}$ and Joe Yuichiro Wakano ${ }^{4,5}$ \\ Accepted to be published in the Journal of Theoretical Biology \\ 4.6.2018
}

1. Department of Mathematics and Statistics, FI-20014 University of Turku, Finland. E-mail: kalle.parvinen@utu.fi

2. Evolution and Ecology Program, International Institute for Applied Systems Analysis (IIASA), A-2361 Laxenburg, Austria

3. Department of Evolutionary Studies of Biosystems, School of Advanced Sciences, SOKENDAI (The Graduate University for Advanced Studies), Shonan Village, Hayama, Kanagawa 240-0193, Japan

4. School of Interdisciplinary Mathematical Sciences, Meiji University, Tokyo 164-8525, Japan

5. Meiji Institute for Advanced Study of Mathematical Sciences, Tokyo 1648525, Japan

Available at https://doi.org/10.1016/j.jtbi.2018.06.005

\begin{abstract}
It is widely recognized that spatial structure in a population has some, and occasionally great, impacts on ecological and evolutionary dynamics. However, it has been observed that in the homogeneous Wright's island model with a certain standard demographic assumption, spatial structure does not affect the fitness gradient of a fecundity-affecting trait. The location and convergence stability of singular strategies thus remain unchanged. Furthermore, evolutionary branching is impossible for small dispersal rates, and for a wide class of fecundity functions, evolutionary branching is impossible for any dispersal rate if branching does not occur in the corresponding well-mixed model. Spatially homogeneous structure thus often inhibits evolutionary branching. Here we study the impact of spatial heterogeneity on evolutionary dynamics. We consider an infinite Wright's island model, where different islands have different capacity and fecundity consequences, and therefore the population is spatially heterogeneous. Through the analysis of metapopulation fitness, we derive its first-order and second-order
\end{abstract}


derivatives with respect to mutant's trait, which are explicitly represented in terms of fecundity derivatives. The selection gradient turns out to be a biased average of local selection pressures in different patch types. We find that evolutionary branching is generally favored in the presence of spatial heterogeneity. We also find a simple condition under which evolutionary branching is particularly favored. Applications to public-goods cooperation and emergent evolutionary branching to cooperators and defectors are discussed.

\section{Highlights:}

- We study trait evolution in a heterogeneous Wright's island model.

- First- and second-order conditions are derived in terms of fecundity derivatives.

- Spatial heterogeneity promotes evolutionary branching.

- Negative correlation between direct benefits to self and others promotes branching.

Keywords: Adaptive dynamics; Cooperation; Evolutionary branching; Natural selection; Dispersal

\section{Introduction}

Adaptive dynamics theory provides us with a general framework to understand evolutionary dynamics based on substitution sequences of biological traits, where mutants repeatedly arise in a population of residents and successful ones eventually take over the population (Dieckmann and Law, 1996; Metz et al., 1996; Geritz et al., 1997, 1998; Wakano and Lehmann, 2014). One of its advantages is that it can predict when disruptive selection occurs, which leads a population into two (and potentially eventually more) distinct subgroups of different phenotypes. Such a phenomenon is called evolutionary branching, and its mathematical condition as well as its application to speciation has been intensively studied (Geritz et al., 1997, 1998; Dieckmann and Doebeli, 1999; Doebeli, 2011).

One of the critical quantities in adaptive dynamics theory is called invasion fitness (Metz et al., 1992), which represents the long-term exponential growth rate of mutants in an environment set by the resident(s). Mutants are deemed successful in invasion if their invasion fitness is positive, otherwise they ultimately go extinct from the population. For structured population models, invasion fitness is occasionally given as the largest eigenvalue of some transition matrix, and may be less tractable than other equivalent measures of invasion success (Lehmann et al., 2016), which are also called fitness proxies. In spatial models of evolution, the metapopulation fitness (Metz and Gyllenberg, 2001; Gyllenberg and Metz, 2001) is a fitness proxy, which measures growth between dispersal generations. A 
mutant disperser may survive dispersal and settle in a spatial patch (sometimes called deme or island). This disperser and all its philopatric descendants form a mutant colony. Metapopulation fitness measures how many mutant dispersers such a colony on average produces during its lifetime. Note that the production of no dispersers, when the original mutant disperser does not survive dispersal and settle, is included in the calculation of the average production.

There is a growing interest in investigating the effect of spatial structure on evolutionary dynamics (Nowak and May, 1992; Nakamaru et al., 1997; van Baalen and Rand, 1998; Frank, 1998; Gandon and Rousset, 1999; Irwin and Taylor, 2001; Parvinen, 2002; Le Galliard et al., 2003; Boots et al., 2004; Hauert and Doebeli, 2004; Rousset, 2004; Ohtsuki et al., 2006; Lion and van Baalen, 2007; Szabó and Fáth., 2007; Taylor et al., 2007; Lehmann et al., 2009; Tarnita et al., 2009; Wild et al., 2009; Allen et al., 2013; Mullon et al., 2018), including the condition of evolutionary branching. Firstly, spatial structure naturally induces more assortment of similar phenotypes than dissimilar ones because of limited dispersal and the resulting spatial segregation (Hamilton, 1964; Fletcher and Doebeli, 2009), and therefore evolutionary dynamics deviate from those predicted for a well-mixed population where individuals are assumed to interact with each other at random (Nowak, 2006; Ohtsuki and Nowak, 2006; Débarre et al., 2014). Secondly, and relevantly to the first point, local competition between similar phenotypes occurs, and this effect is often termed as local kin competition (Taylor, 1992a,b; Wilson et al., 1992; Gandon and Michalakis, 1999; Rousset and Ronce, 2004; Lehmann and Keller, 2006). For those reasons, evolution of traits proceeds differently in a spatially structured population than in a well-mixed population. One interesting exception is known as Taylor's cancellation result (Taylor, 1992a,b), in which the effect of helping kin is completely cancelled out by increased local kin competition and monomorphic evolution proceeds as if social effect on neighbors did not exist.

It is also known that the condition of evolutionary branching can be affected by the spatial structure of the population (Day, 2000, 2001; Ajar, 2003; Doebeli and Dieckmann, 2003; Nurmi and Parvinen, 2008; Haller et al., 2013; Wakano and Lehmann, 2014; Mullon et al., 2016). One factor that could cause disruptive selection is such spatial heterogeneity in which a certain trait is favored in one patch type but disfavored in another patch type. In such case, a generalist ESS could be destabilized, leading to evolutionary branching (Meszéna et al., 1997; Sasaki and de Jong, 1999; Débarre and Gandon, 2011). Day (2000) found that in a resource competition model spatial heterogeneity generates disruptive selection when the migration rate is low, but when the migration is high it generates either disruptive or stabilizing selection depending on detailed demographic conditions. Doebeli and Dieckmann (2003) and Haller et al. (2013) found that an intermediate level of spatial heterogeneity maximizes the propensity of diversification. Nurmi and Parvinen (2008) studied the evolution of specialization in a spatially heterogeneous metapopulation model, in which environmental heterogeneity favors evolutionary branching.

Another effect of spatial structure on evolutionary branching is the division 
of population into patches with potentially finite local population sizes. Several analytic studies exist in case of homogeneous spatial models where all local populations have the same fixed finite size. Ajar (2003) studied Wright's island model and derived the second-order derivative of metapopulation fitness $R_{\mathrm{m}}$ in a general expression. Wakano and Lehmann (2014) took a so-called trait-distribution approach and derived the dynamics of trait variance to predict whether evolutionary branching occurs or not. Mullon et al. (2016) and Mullon et al. (2018) studied the joint evolution of multiple traits, and showed conditions for evolutionary branching to non-dispersing cooperators and dispersing defectors. Parvinen et al. (2017) assumed that individual traits affect their fecundity in Wright's island model and derived an explicit condition in terms of derivatives of fecundity functions. Furthermore, they showed a sufficient condition under which evolutionary branching does not occur in Wright's island model given that it does not occur in a corresponding well-mixed model. They also showed that when either the migration rate or the survival probability in dispersal is sufficiently close to zero, evolutionary branching never occurs.

Here we extend the model of Parvinen et al. (2017) to incorporate spatial heterogeneity. In our new model, there are infinitely many patches with finite local populations that are connected via migration, as in the usual Wright's model, but there are different patch types and those patches differ in local population size as well as in the fecundity consequences of interactions between individuals. Heterogeneous populations have been studied less intensively than homogeneous populations under such setting. Parvinen (2002) studied evolutionary branching of dispersal strategies in a heterogeneous metapopulation model with different patch types. In contrast with our model, Parvinen (2002) studied large local populations, in which local population densities are not fixed, but change in time due to local population growth, emigration, immigration and catastrophes. Rodrigues and Gardner (2012) studied spatio-temporal heterogeneity of patch resource availability and its impact on evolution of helping and harming. They assumed two types of patches, high-quality and low-quality ones, and found that facultative helping and harming are both favored by natural selection when both spatial and temporal heterogeneity of patch quality exist at the same time. Introducing spatial heterogeneity alone does not change Taylor's cancellation result, although their result is limited because they considered only two types of patches with the same finite size.

The aim of our paper is to clarify the conditions of evolutionary singularity and evolutionary branching in the heterogeneous island model with non-overlapping generations in terms of fecundity derivatives, and to apply these results to concrete examples. We consider spatial heterogeneity but not temporal heterogeneity. We will show that under the existence of heterogeneity in patch types evolutionary branching is generally favored more than in the homogeneous case, especially when the fecundity derivative with respect to one's strategy is negatively correlated to that with respect to other's strategy. In other words, evolutionary branching is especially favored, when in some patch types an individual that increases its 
strategy value increases its own fecundity and decreases the fecundity of others, and in some other patch types decreasing the strategy value has a similar effect. As an example we discuss public-goods cooperation and show that evolutionary branching of the trait that represents the amount of contribution to public-goods occurs when there is significant spatial heterogeneity.

This paper is structured as follows. In section 2 we describe model assumptions. In section 3 we provide our analytical results. In section 4 we discuss an application of our analytical results to an example of public-goods cooperation. In section 5 , we conclude our paper with a discussion.

\section{Model description and metapopulation fitness}

\subsection{Multitype island model and fecundity function}

Similar to our previous work (Parvinen et al., 2017), we consider an extended version of Wright's island model (Wright, 1931), which consists of infinitely many habitat patches (demes). Here we include the possibility for spatial heterogeneity, so that patches can be of $N$ different types. The proportion of patches of type $k$ is $\pi_{k}$, and naturally

$$
\sum_{k=1}^{N} \pi_{k}=1
$$

holds.

In the beginning of the season each patch of type $k$ contains $n_{k}$ adult individuals. These adults may differ in their reproductive strategies $s$, which affect their fecundity $\gamma F_{k}$ that represents the number of juveniles that they produce. Note that without loss of generality, $\gamma$ can be assumed to be the same among all patch types. Throughout the manuscript, $\gamma$ is considered to be very large (actually $\gamma \rightarrow \infty)$. More precisely, the relative fecundity for an adult with strategy $s_{1}$, when the strategies of the other individuals are $\boldsymbol{s}_{n_{k}-1}=\left(s_{2}, \ldots, s_{n_{k}}\right)$ is

$$
F_{k}\left(s_{1} ; \boldsymbol{s}_{n_{k}-1}\right)=F_{k}\left(s_{1} ;\left(s_{2}, \ldots, s_{n_{k}}\right)\right) .
$$

Because the order of strategies in the vector $\boldsymbol{s}_{n_{k}-1}$ does not affect fecundity, some symmetry properties naturally arise; such that $F_{k}\left(s_{1} ; \boldsymbol{s}_{n_{k}-1}\right)$ remains the same value for any permutation of the components of $\boldsymbol{s}_{n_{k}-1}$.

The emigration probability $m, 0<m \leqslant 1$, is the proportion of juveniles that will disperse. The proportion $p, 0<p \leqslant 1$ of dispersed juveniles will survive dispersal and land in a random patch, so that the probability to arrive in a patch of type $k$ is $\pi_{k}$. The other juveniles die during dispersal. The present adults are assumed not to survive until the next season. The local adult population size is assumed to be fixed, so that the $n_{k}$ individuals to become adults in a patch of type $k$ in the next season are randomly chosen among the juveniles in each patch after immigration. 
Next we study the invasion potential of a mutant with strategy $s_{\text {mut }}$ in an environment set by a resident with strategy $s_{\text {res }}$. The relative fecundity of a resident, when there are $i$ mutants and $n_{k}-i$ residents (including the focal resident) in the same patch is

$$
F_{k, \text { res }}^{i}=F_{k, \text { res }}^{i}\left(s_{\text {res }}, s_{\text {mut }}\right)=F_{k}\left(s_{\text {res }} ;(\underbrace{s_{\text {mut }}, \ldots, s_{\text {mut }}}_{\#=i}, \underbrace{s_{\text {res }}, \ldots, s_{\text {res }}}_{\#=n_{k}-i-1})\right) .
$$

Analogously, the relative fecundity of a mutant, when there are $i$ mutants (including the focal mutant) and $n_{k}-i$ residents in the same patch, is

$$
F_{k, \text { mut }}^{i}=F_{k, \text { mut }}^{i}\left(s_{\text {res }}, s_{\text {mut }}\right)=F_{k}\left(s_{\text {mut }} ;(\underbrace{s_{\text {mut }}, \ldots, s_{\text {mut }}}_{\#=i-1}, \underbrace{s_{\text {res }}, \ldots, s_{\text {res }}}_{\#=n_{k}-i})\right) .
$$

In particular,

$$
F_{k, \text { res }}^{0}=F_{k}\left(s_{\text {res }},\left(s_{\text {res }}, \ldots, s_{\text {res }}\right)\right) .
$$

is the relative fecundity of a resident, when all individuals in the same patch are residents.

\subsection{The metapopulation fitness, $R_{\mathrm{m}}$}

The metapopulation reproduction number (metapopulation fitness) is the expected number of dispersing mutant juveniles that are produced by the mutant colony of one dispersing mutant juvenile (Gyllenberg and Metz, 2001; Metz and Gyllenberg, 2001). The mutant colony may also be empty, if the original mutant disperser does not survive dispersal and settle as an adult at the destination patch, and this case is included when the expected value is calculated. Analogous to Parvinen (2002), here we show how to extend the spatially homogeneous case (Ajar, 2003; Parvinen et al., 2017) into the spatially heterogeneous situation. Although we do not consider evolution of dispersal here, the following expressions are formulated for a mutant potentially differing from the resident in its dispersal behavior, in addition to the reproductive behaviour. Dispersal probabilities of residents and mutants are denoted by $m_{\text {res }}$ and $m_{\text {mut }}$, respectively.

\subsubsection{Settlement probability}

Assume that residents are dominant in number in the metapopulation, and consider a mutant juvenile that has just emigrated from a patch. For it to be an adult in the next generation, it has to survive dispersal (which occurs with probability $p$ ) and then has to settle successfully in a patch. Let us consider the latter probability. 
The focal mutant juvenile will land in a patch of type $k$ with probability $\pi_{k}$. In this patch it will compete with resident immigrants and natal residents. The amount of resident immigrants arriving at this patch is

$$
p m_{\mathrm{res}} \sum_{l=1}^{N} \pi_{l} \underbrace{n_{l} \gamma F_{l, \mathrm{res}}^{0}}_{\begin{array}{c}
\text { \# of juveniles produced } \\
\text { from a patch of type } l
\end{array}}=\gamma \cdot \underbrace{p m_{\mathrm{res}} \underbrace{\sum_{l=1}^{N} \pi_{l} n_{l} F_{l, \mathrm{res}}^{0}}_{\equiv\langle n F\rangle}}_{\equiv I_{\mathrm{res}}}=\gamma I_{\mathrm{res}},
$$

where

$$
\langle n F\rangle=\sum_{l=1}^{N} \pi_{l} n_{l} F_{l, \text { res }}^{0}
$$

is the average reproductive potential of patches. The amount of resident immigrants, $\gamma I_{\text {res }}$, is independent of the type of the patch, whereas the amount of natal residents in a patch of type $k,\left(1-m_{\text {res }}\right) n_{k} F_{k \text {,res }}^{0}$, does depend on the patch type $k$. For the focal mutant immigrant, therefore, its proportion of the whole amount of juveniles in this patch is approximately

$$
q_{k}=\frac{1}{n_{k} \gamma F_{k, \text { res }}^{0}\left(1-m_{\mathrm{res}}\right)+\gamma I_{\mathrm{res}}+1} .
$$

Remember that $n_{k}$ juveniles become adults in a patch of type $k$. The probability that the focal mutant will be among the $n_{k}$ juveniles chosen to be adults in the patch is (for large $\gamma$ )

$$
P_{k}(\text { settlement })=q_{k}+\left(1-q_{k}\right) q_{k}+\ldots+\left(1-q_{k}\right)^{n_{k}-1} q_{k}=1-\left(1-q_{k}\right)^{n_{k}} \approx n_{k} q_{k} .
$$

From these calculations, the probability with which a given mutant juvenile that has just emigrated from its natal patch survives dispersal and becomes an adult in a patch of type $k$ is given by $p P_{k}$ (settlement).

\subsubsection{Dynamics of the mutant colony}

The initial mutant immigrant and all its descendants (if any), as long as they stay in the focal patch, form a mutant colony. Now we investigate the dynamics of such a colony in a patch of type $k$. In case there are currently $i$ adult mutants in the patch, there are $n_{k}-i$ resident adults. These adults will get offspring, so that the proportion of mutant juveniles competing in this patch in the next generation is

$$
\begin{aligned}
p_{k, i} & =\frac{\left(1-m_{\mathrm{mut}}\right) i \gamma F_{k, \text { mut }}^{i}}{\left(1-m_{\mathrm{mut}}\right) i \gamma F_{k, \text { mut }}^{i}+\left(1-m_{\mathrm{res}}\right)\left(n_{k}-i\right) \gamma F_{k, \mathrm{res}}^{i}+\gamma I_{\mathrm{res}}} \\
& =\frac{\left(1-m_{\mathrm{mut}}\right) i F_{k, \text { mut }}^{i}}{\left(1-m_{\mathrm{mut}}\right) i F_{k, \mathrm{mut}}^{i}+\left(1-m_{\mathrm{res}}\right)\left(n_{k}-i\right) F_{k, \text { res }}^{i}+I_{\mathrm{res}}} .
\end{aligned}
$$


Note that the $\gamma$ terms cancel each other.

The number of adult mutants in the next generation follows a binomial probability distribution, so that the probability that there will be $j$ adult mutants in this patch in the next generation is

$$
t_{k, j i}=\left(\begin{array}{c}
n_{k} \\
j
\end{array}\right)\left(p_{k, i}\right)^{j}\left(1-p_{k, i}\right)^{n_{k}-j} .
$$

We collect these values into the transition matrix $\mathbf{T}_{k}=\left(t_{k, j i}\right)$ where $i, j=1, \ldots, n_{k}$. The transition matrices in different patch types are different, and have even different dimensions, if $n_{k}$ are different. The absorbing state $i=0$ is on purpose left away. The probability distribution of adult mutants in this patch at time $t$ satisfies the recursion

$$
\alpha_{k}(t)=\left(\mathbf{T}_{k}\right)^{t} \alpha_{k, 0}, \text { where } \alpha_{k, 0}=\{1, \underbrace{0, \ldots, 0}_{n_{k}-1}\}^{T},
$$

where the symbol ${ }^{T}$ represents matrix transposition.

\subsubsection{Disperser production}

During the lifetime of the mutant colony, some mutant juveniles will emigrate. We want to calculate their expected amount. When the size of the mutant colony is $i$ in a patch of type $k$, the expected amount of emigrating juveniles is $i m_{\text {mut }} \gamma F_{k \text {,mut }}(i)$. These values are multiplied with $p P_{k}$ (settlement), i.e., the probability that the initial dispersing mutant juvenile survived dispersal and settled as an adult, provided that it arrived in a patch of type $k$. These values are collected into a column vector

$$
\begin{aligned}
E_{k} & =p P_{k} \text { (settlement) } m_{\text {mut }} \gamma\left\{F_{k, \text { mut }}^{1}, 2 F_{k, \text { mut }}^{2}, \ldots, n_{k} F_{k, \text { mut }}^{n_{k}}\right\}^{T} \\
& =\frac{p m_{\text {mut }} n_{k}}{\left(1-m_{\text {res }}\right) n_{k} F_{k, \text { res }}+I_{\text {res }}}\left\{F_{k, \text { mut }}^{1}, 2 F_{k, \text { mut }}^{2}, \ldots, n_{k} F_{k, \text { mut }}^{n_{k}}\right\}^{T} \\
& =\frac{p m_{\text {mut }} n_{k}}{I_{\text {res }}} d_{k}\left\{F_{k, \text { mut }}^{1}, 2 F_{k, \text { mut }}^{2}, \ldots, n_{k} F_{k, \text { mut }}^{n_{k}}\right\}^{T},
\end{aligned}
$$

where the second equality holds because $\gamma$ is large. The expression $d_{k}$ is the backward migration probability, discussed more around equation (3.1).

We denote by $\omega_{k}$ the sojourn time vector. The component $\omega_{k, i}$ represents the expected number of times that a mutant colony in patch of type $k$ will have size $i$ during its lifetime. Based on the dynamics of the mutant colony we have

$$
\omega_{k}=\sum_{t=0}^{\infty} \alpha_{k}(t)=\sum_{t=0}^{\infty}\left(\mathbf{T}_{k}\right)^{t} \alpha_{k, 0}=\alpha_{k, 0}+\mathbf{T}_{k} \sum_{t=0}^{\infty}\left(\mathbf{T}_{k}\right)^{t} \alpha_{k, 0}=\alpha_{k, 0}+\mathbf{T}_{k} \omega_{k},
$$

from which we furthermore obtain

$$
\left(\mathbf{I}-\mathbf{T}_{k}\right) \omega_{k}=\alpha_{k, 0} \Rightarrow \omega_{k}=\left(\mathbf{I}-\mathbf{T}_{k}\right)^{-1} \alpha_{k, 0}
$$


The sojourn time vector $\omega_{k}$ is thus obtained by solving a system of linear equations, but there are also other methods to calculate it numerically, such as the limit of the recurrence $\omega_{k}(t+1)=\mathbf{T}_{k} \omega_{k}(t)+\alpha_{k, 0}$, for any initial condition.

Now the expected number of dispersing mutant juveniles that are produced by the mutant colony of one dispersing mutant juvenile, provided that it arrived in a patch of type $k$, is $E_{k}^{T} \omega_{k}$. Analogously to Parvinen (2002), we need to calculate the expectation over the patch type distribution to obtain the metapopulation reproduction ratio (Gyllenberg and Metz, 2001; Metz and Gyllenberg, 2001)

$$
R_{\mathrm{m}}=\sum_{k=1}^{N} \pi_{k} E_{k}^{T} \omega_{k} .
$$

This metapopulation reproduction ratio (metapopulation reproductive number, metapopulation fitness) is sign-equivalent with invasion fitness $r\left(R_{\mathrm{m}}>1\right.$ if and only if $r>0$ ), and other measures of mutant's invasion success in metapopulations (Metz and Leimar, 2011; Lehmann et al., 2016).

\section{$3 \quad$ Evolution of the fecundity-affecting strategy $s$}

From now on, we only consider fecundity-affecting strategies, so that $m_{\text {res }}=$ $m_{\text {mut }}=m$. For the spatially homogeneous case Parvinen et al. (2017) presented explicit expressions for the fitness gradient and second derivatives with respect to the fecundity-affecting strategy $s$, and investigated the effect of spatial structure on evolutionary branching. Here we extend these investigations into the spatially heterogeneous case. Table 1 in the end of this section summarizes results and differences between the homogeneous and heterogeneous cases.

According to (2.15), the vector $\omega_{k}$ needed in the calculation of metapopulation fitness (2.16) is obtained by solving a system of linear equations. In order to calculate the fitness gradient and other relevant derivatives, we need to calculate the derivatives $\omega_{k}^{\prime}=\left.\frac{\partial}{\partial s_{\mathrm{mut}}} \omega_{k}\right|_{s_{\mathrm{mut}}=s_{\mathrm{res}}}$ and $\omega_{k}^{\prime \prime}=\left.\frac{\partial^{2}}{\partial s_{\mathrm{mut}}^{2}} \omega_{k}\right|_{s_{\mathrm{mut}}=s_{\mathrm{res}}}$. Following Parvinen et al. (2017), they can be obtained by using the implicit function theorem. Then by taking advantage of symmetry properties of the fecundity functions, we obtain the explicit expressions presented below in Theorems 1 and 3. The calculations are mostly straightforward generalizations of the corresponding homogeneous results by Parvinen et al. (2017). Below we only explain how the heterogeneous results differ from the homogeneous ones, but for the sake of completeness, we present the complete proofs in the Electronic supplementary material (Appendix C).

The backward migration probability $d_{k}$, i.e., the proportion of adults that are immigrant in a monomorphic population in a patch of type $k$ depends on the amount of immigrants $I_{\text {res }}$, and on the local number of adults $n_{k}$ and on local fecundity $F_{k, \text { res }}^{0}$ as 


$$
d_{k}=\frac{I_{\mathrm{res}}}{(1-m) n_{k} F_{k, \mathrm{res}}^{0}+I_{\mathrm{res}}}=\frac{m p\langle n F\rangle}{(1-m) n_{k} F_{k, \mathrm{res}}^{0}+m p\langle n F\rangle}=\frac{m p}{(1-m) V_{k}+m p},
$$

where

$$
V_{k}=\frac{n_{k} F_{k, \text { res }}^{0}}{\langle n F\rangle}=\frac{n_{k} F_{k, \text { res }}^{0}}{\sum_{l=1}^{N} \pi_{l} n_{l} F_{l, \text { res }}^{0}}
$$

is the relative reproductive potential of a patch of type $k$. Note that $V_{k}$ is different from the reproductive value (Taylor, 1990). In fact, we show in Appendix B that the "individual" reproductive value of an adult in a patch of type $k$ is proportional to $F_{k \text {,res }}^{0} / d_{k}$, and therefore the "patch" reproductive value of a patch of type $k$ is proportional to $n_{k} F_{k \text {,res }}^{0} / d_{k}$, which is qualitatively different from $V_{k}$ by the factor $1 / d_{k}$ (after neglecting a normalization constant). Note that if the product $n_{k} F_{k \text {,res }}^{0}$ is the same in all patch types, we have $V_{k}=1$ for all $k$, and the backward migration probability becomes $d=p m /(1-m+p m)$, which is the same as Eq. (3.5) of Parvinen et al. (2017).

By using a simple argument (for details, see the Electronic supplementary material (Appendix C)), we can formally prove that most of the results derived for a homogeneous population in Parvinen et al. (2017) are directly applicable to our heterogeneous model; we should use $d_{k}$ and $n_{k}$ in their results instead of $d$ and $n$. More specifically, the first-order derivative of the metapopulation fitness in our model, $R_{\mathrm{m}}$, with respect to mutant's strategy, $s_{\mathrm{mut}}$, turns out to be given by the weighted sum of Eq. (3.4) in Parvinen et al. (2017) ( $d$ and $n$ there being replaced with $d_{k}$ and $n_{k}$ ), where the appropriate weight should be $\pi_{k} V_{k}$. Similarly, the second-order derivative is given as the weighted sum of Eq. (3.10) in Parvinen et al. (2017) ( $d$ and $n$ there being replaced with $d_{k}$ and $n_{k}$ ) with the same weight, $\pi_{k} V_{k}$.

\subsection{Fitness gradient}

Because the numbering of other individuals in a patch is arbitrary, the fecundity function $F_{k}\left(s_{1} ;\left(s_{2}, \ldots, s_{n_{k}}\right)\right)$ has symmetry properties, so that it only has two different first-order derivatives. One is the first-order derivative with respect to the strategy of self (subscript "S"), which is

$$
F_{k, \mathrm{~S}}=\left.\frac{\partial}{\partial s_{1}} F_{k}\left(s_{1} ;\left(s_{2}, \ldots, s_{n_{k}}\right)\right)\right|_{s_{1}=\cdots=s_{n_{k}}=s_{\mathrm{res}}} .
$$

The other first-order derivative is that with respect to the strategy of anybody else in the patch (subscript "D" for "Different")

$$
F_{k, \mathrm{D}}=\left.\frac{\partial}{\partial s_{j}} F_{k}\left(s_{1} ;\left(s_{2}, \ldots, s_{n_{k}}\right)\right)\right|_{s_{1}=\cdots=s_{n_{k}}=s_{\mathrm{res}}}, \text { where } j \in\left\{2, \ldots, n_{k}\right\}
$$


The right-hand side of that equality is independent of the choice of $j$.

Theorem 1. The selection gradient of a fecundity-affecting strategy is

$$
\begin{aligned}
D_{1}\left(s_{\mathrm{res}}\right) & =\left.\frac{\partial}{\partial s_{\mathrm{mut}}} R_{\mathrm{m}}\right|_{s_{\mathrm{mut}}=s_{\mathrm{res}}}=\sum_{k=1}^{N} \pi_{k} V_{k}\left\{\frac{n_{k}\left(2-d_{k}\right)}{n_{k}-\left(n_{k}-1\right)\left(1-d_{k}\right)^{2}} \frac{F_{k, \mathrm{~S}}}{F_{k, \mathrm{res}}^{0}}\right\} \\
& =\frac{1}{\langle n F\rangle} \sum_{k=1}^{N} \pi_{k} \frac{n_{k}^{2}\left(2-d_{k}\right)}{n_{k}-\left(n_{k}-1\right)\left(1-d_{k}\right)^{2}} F_{k, \mathrm{~S}} .
\end{aligned}
$$

Proof. Straightforward generalization of Theorem 1 of Parvinen et al. (2017). See the Electronic supplementary material (Appendix C) for details.

The fitness gradient is thus a biased sum of local fecundity derivatives with respect to self, $F_{k, \mathrm{~S}}$. As in the spatially homogeneous case, the fecundity derivative(s) with respect to other, $F_{k, \mathrm{D}}$, do not appear in the fitness gradient. This result can be seen as an extension of Taylor's cancellation result (Taylor, 1992a; Taylor and Irwin, 2000). One consequence of this result is that even under spatial heterogeneity altruistic cooperation cannot evolve in the Wright's island model under the demography assumed here.

This result connects to Rodrigues and Gardner (2012) in the following way. A special case, $N=2, n_{1}=n_{2}=n, F_{1, \mathrm{~S}}=-C, F_{2}\left(s_{1}, \ldots, s_{n}\right)=c F_{1}\left(s_{1}, \ldots, s_{n}\right)$, yields their Result 1 (obligate helping and harming), where a constant $0<c<1$ represents low quality of patch type 2 . Another special case, $N=2, n_{1}=n_{2}=$ $n, F_{1, \mathrm{~S}}=-C, F_{2}\left(s_{1}, \ldots, s_{n}\right)=$ const., yields their Result 2 (facultative helping and harming).

\subsection{Convergence stability}

Similarly to before, by using the property of the fecundity function, $F_{k}\left(s_{1} ;\left(s_{2}, \ldots, s_{n_{k}}\right)\right.$ ), that the order of the other strategies than $s_{1}$ can be freely permutated, we see that there are only four kinds of second-order derivatives of $F_{k}$ :

$$
\begin{aligned}
F_{k, \mathrm{SS}} & =\left.\frac{\partial^{2}}{\partial s_{1}^{2}} F_{k}\left(s_{1} ;\left(s_{2}, \ldots, s_{n}\right)\right)\right|_{s_{1}=\cdots=s_{n}=s_{\mathrm{res}}}, \text { where } j \in\{2, \ldots, n\} \\
F_{k, \mathrm{DD}} & =\left.\frac{\partial^{2}}{\partial s_{j}^{2}} F_{k}\left(s_{1} ;\left(s_{2}, \ldots, s_{n}\right)\right)\right|_{s_{1}=\cdots=s_{n}=s_{\mathrm{res}}}, \text { where } j \in\{2, \ldots, n\} \\
F_{k, \mathrm{SD}} & =\left.\frac{\partial^{2}}{\partial s_{1} \partial s_{j}} F_{k}\left(s_{1} ;\left(s_{2}, \ldots, s_{n}\right)\right)\right|_{s_{1}=\cdots=s_{n}=s_{\mathrm{res}}}, \text { where } j, l \in\{2, \ldots, n\}, j \neq l . \\
F_{k, \mathrm{DD}^{\prime}} & =\left.\frac{\partial^{2}}{\partial s_{j} \partial s_{l}} F_{k}\left(s_{1} ;\left(s_{2}, \ldots, s_{n}\right)\right)\right|_{s_{1}=\cdots=s_{n}=s_{\mathrm{res}}}
\end{aligned}
$$

For singular strategies the fitness gradient is zero, $D_{1}\left(s^{*}\right)=0$. A singular strategy is convergence stable (evolutionarily attracting), if the fitness gradient 
is a decreasing function of $s_{\text {res }}$ at the singularity, i.e., the derivative $D_{1}^{\prime}\left(s_{\text {res }}\right)=$ $\frac{\mathrm{d}}{\mathrm{d} s_{\mathrm{res}}} D_{1}\left(s_{\mathrm{res}}\right)$ is negative at the singular strategy.

Theorem 2. The expression $D_{1}^{\prime}\left(s^{*}\right)$ for a singular strategy $s^{*}$ is

$$
\begin{aligned}
D_{1}^{\prime}\left(s^{*}\right)=\frac{1}{\langle n F\rangle} & {\left[\sum_{k=1}^{N} \pi_{k} \frac{n_{k}^{2}\left(2-d_{k}\right)}{n_{k}-\left(n_{k}-1\right)\left(1-d_{k}\right)^{2}}\left(F_{k, \mathrm{SS}}+\left(n_{k}-1\right) F_{k, \mathrm{SD}}\right)\right.} \\
& \left.-\sum_{k=1}^{N} \pi_{k} \frac{n_{k}^{2}\left(n_{k}+\left(n_{k}-1\right)\left(1-d_{k}\right)\left(3-d_{k}\right)\right)}{\left(n_{k}-\left(n_{k}-1\right)\left(1-d_{k}\right)^{2}\right)^{2}}\left(\frac{\mathrm{d}}{\mathrm{d} s_{\mathrm{res}}} d_{k}\right) F_{k, \mathrm{~S}}\right],
\end{aligned}
$$

where

$$
\frac{\mathrm{d}}{\mathrm{d} s_{\mathrm{res}}} d_{k}=-d_{k}\left(1-d_{k}\right) \frac{1}{V_{k}}\left(\frac{\mathrm{d}}{\mathrm{d} s_{\mathrm{res}}} V_{k}\right)
$$

and

$$
\frac{\mathrm{d}}{\mathrm{d} s_{\mathrm{res}}} V_{k}=\frac{1}{\langle n F\rangle}\left[n_{k}\left(F_{k, \mathrm{~S}}+\left(n_{k}-1\right) F_{k, \mathrm{D}}\right)-n_{k} F_{k, \text { res }}^{0} \frac{\left\langle n\left(F_{\mathrm{S}}+(n-1) F_{\mathrm{D}}\right)\right\rangle}{\langle n F\rangle}\right] .
$$

Proof. Differentiation of the second row of (3.5), taking into account that $D_{1}\left(s^{*}\right)=$ 0 .

\subsection{Evolutionary stability}

Theorem 3. The second-order derivative with respect to a fecundity-affecting strategy $s$ can be written as

$$
\begin{aligned}
& D_{2}\left(s_{\mathrm{res}}\right)=\left.\frac{\partial^{2}}{\partial s_{\mathrm{mut}}^{2}} R_{\mathrm{m}}\right|_{s_{\mathrm{mut}}=s_{\mathrm{res}}} \\
&=\sum_{k=1}^{N} \pi_{k} V_{k}\left\{C _ { k } \left[\phi_{k, \mathrm{SS}}\left(\frac{F_{k, \mathrm{SS}}}{F_{k, \mathrm{res}}^{0}}\right)+\phi_{k, \mathrm{SD}}\left(\frac{F_{k, \mathrm{SD}}}{F_{k, \mathrm{res}}^{0}}\right)+\phi_{k, \mathrm{DD}^{\prime}}\left(\frac{F_{k, \mathrm{DD}^{\prime}}}{F_{k, \mathrm{res}}^{0}}\right)\right.\right. \\
&\left.\left.\quad+\psi_{k, \mathrm{~S} \times \mathrm{S}}\left(\frac{F_{k, \mathrm{~S}}}{F_{k, \mathrm{res}}^{0}}\right)^{2}+\psi_{k, \mathrm{~S} \times \mathrm{D}}\left(\frac{F_{k, \mathrm{~S}}}{F_{k, \mathrm{res}}^{0}}\right)\left(\frac{F_{k, \mathrm{D}}}{F_{k, \mathrm{res}}^{0}}\right)+\psi_{k, \mathrm{D} \times \mathrm{D}}\left(\frac{F_{k, \mathrm{D}}}{F_{k, \mathrm{res}}^{0}}\right)^{2}\right]\right\},
\end{aligned}
$$

where

$$
C_{k}=\frac{n_{k}}{\left\{n_{k}-\left(n_{k}-1\right)\left(1-d_{k}\right)^{2}\right\}^{2}\left\{n_{k}^{2}-\left(n_{k}-1\right)\left(n_{k}-2\right)\left(1-d_{k}\right)^{3}\right\}}>0
$$


and

$$
\begin{aligned}
& \phi_{k, \mathrm{SS}}=\left(2-d_{k}\right)\left\{n_{k}-\left(n_{k}-1\right)\left(1-d_{k}\right)^{2}\right\}\left\{n_{k}^{2}-\left(n_{k}-1\right)\left(n_{k}-2\right)\left(1-d_{k}\right)^{3}\right\}>0 \\
& \phi_{k, \mathrm{SD}}= 2\left(n_{k}-1\right)\left(1-d_{k}\right)^{2}\left\{n_{k}-\left(n_{k}-1\right)\left(1-d_{k}\right)^{2}\right\}\left\{n_{k}+n_{k}\left(1-d_{k}\right)+\left(n_{k}-2\right)\left(1-d_{k}\right)^{2}\right\} \geqslant 0 \\
& \phi_{k, \mathrm{DD}}=\left(n_{k}-1\right)\left(n_{k}-2\right) d_{k}\left(1-d_{k}\right)^{3}\left\{n_{k}-\left(n_{k}-1\right)\left(1-d_{k}\right)^{2}\right\} \geqslant 0 \\
& \psi_{k, \mathrm{~S} \times \mathrm{S}}= 2\left(1-d_{k}\right)\left\{n_{k}^{3}+2 n_{k}^{2}\left(n_{k}-1\right)\left(1-d_{k}\right)+n_{k}\left(n_{k}-1\right)^{2}\left(1-d_{k}\right)^{2}-n_{k}^{2}\left(n_{k}-1\right)\left(1-d_{k}\right)^{3}\right. \\
&\left.\quad-\left(2 n_{k}^{3}-6 n_{k}^{2}+5 n_{k}-1\right)\left(1-d_{k}\right)^{4}-\left(n_{k}-1\right)^{3}\left(1-d_{k}\right)^{5}\right\} \geqslant 0 \\
& \psi_{k, \mathrm{~S} \times \mathrm{D}}=-2\left(n_{k}-1\right)\left(1-d_{k}\right)^{4}\left\{n_{k}+2\left(n_{k}-1\right)\left(1-d_{k}\right)^{2}\right\} \leqslant 0 \\
& \psi_{k, \mathrm{D} \times \mathrm{D}}=-2\left(n_{k}-1\right)^{2} d_{k}\left(1-d_{k}\right)^{3}\left\{n_{k}-\left(n_{k}-1\right)\left(1-d_{k}\right)^{2}\right\} \leqslant 0 .
\end{aligned}
$$

The second-order derivative can also be written as

$$
\begin{aligned}
\sum_{k=1}^{N} \pi_{k} V_{k}\left\{C _ { k } \left[\xi_{k, \mathrm{SS}}\left(\frac{F_{k, \mathrm{SS}}}{F_{k, \mathrm{res}}^{0}}\right)+\frac{\phi_{k, \mathrm{SD}}}{n_{k}-1}\left(\frac{F_{k, \mathrm{SS}}+\left(n_{k}-1\right) F_{k, \mathrm{SD}}}{F_{k, \mathrm{res}}^{0}}\right)+\phi_{k, \mathrm{DD}^{\prime}}\left(\frac{F_{k, \mathrm{DD}^{\prime}}}{F_{k, \mathrm{res}}^{0}}\right)\right.\right. \\
\left.\left.+\psi_{k, \mathrm{~S} \times \mathrm{S}}\left(\frac{F_{k, \mathrm{~S}}}{F_{k, \mathrm{res}}^{0}}\right)^{2}+\psi_{k, \mathrm{~S} \times \mathrm{D}}\left(\frac{F_{k, \mathrm{~S}}}{F_{k, \mathrm{res}}^{0}}\right)\left(\frac{F_{k, \mathrm{D}}}{F_{k, \mathrm{res}}^{0}}\right)+\psi_{k, \mathrm{D} \times \mathrm{D}}\left(\frac{F_{k, \mathrm{D}}}{F_{k, \text { res }}^{0}}\right)^{2}\right]\right\},
\end{aligned}
$$

where

$$
\begin{aligned}
\xi_{k, \mathrm{SS}}= & \phi_{k, \mathrm{SS}}-\frac{\phi_{k, \mathrm{SD}}}{n_{k}-1} \\
= & d_{k}\left[n_{k}^{2}+2\left(1-d_{k}\right) n_{k}^{2}+2\left(1-d_{k}\right)^{2} n_{k}\left(n_{k}-1\right)+\left(1-d_{k}\right)^{3}\left(n_{k}-2\right)\left(n_{k}+1\right)\right] \\
& \times\left\{n_{k}-\left(n_{k}-1\right)\left(1-d_{k}\right)^{2}\right\} \geqslant 0 .
\end{aligned}
$$

Proof. Straightforward generalization of Theorem 3 of Parvinen et al. (2017). See the Electronic supplementary material (Appendix C) for details.

The coefficients in (3.12) have descriptive notation. In (3.10), the coefficients $\phi_{k, \mathrm{SS}}, \phi_{k, \mathrm{SD}}, \phi_{k, \mathrm{DD}^{\prime}}$ are multiplied with the corresponding second-order derivatives of the fecundity function, whereas the coefficients $\psi_{k, \mathrm{~S} \times \mathrm{S}}, \psi_{k, \mathrm{~S} \times \mathrm{D}}$ and $\psi_{k, \mathrm{D} \times \mathrm{D}}$ are multiplied with the corresponding two first-order derivatives of the fecundity function.

Figure 1 illustrates the coefficients of $\left[E_{k}^{T} \omega_{k}\right]^{\prime \prime}$ given by equation (3.13) as a function of $d_{k}$ for two values of $n_{k}$. Only three coefficients are nonzero for $d_{k}=0$ and just one coefficient is nonzero for $d_{k}=1$. These properties will be used in Section 3.4. Many of the curves are non-monotonic, so that non-monotonic effects of dispersal on evolutionary branching may be possible. 
a)

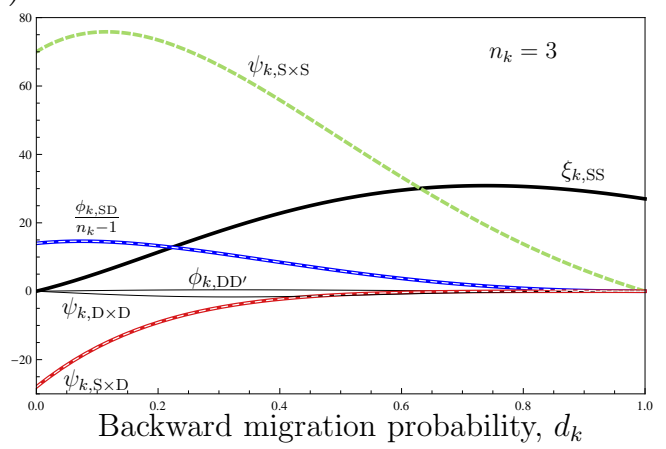

b)

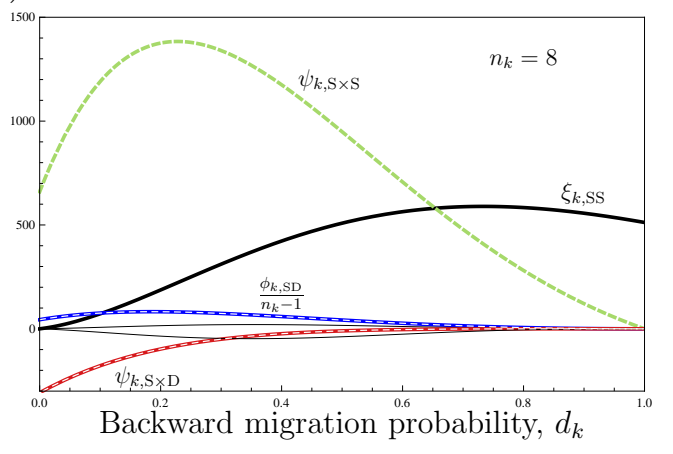

Figure 1: Coefficients of $\left[E_{k}^{T} \omega_{k}\right]^{\prime \prime}$ given by equation (3.13) as a function of $d_{k}$ when (a) $n_{k}=3$ and (b) $n_{k}=8$. The three dashed curves correspond to coefficients that are nonzero at $d_{k}=0$. The solid thick black curve corresponds to $\xi_{k, \mathrm{SS}}$, the only nonzero coefficient at $d_{k}=1$.

\subsection{Spatial heterogeneity can promote evolutionary branch- ing}

General analytical investigation of (3.7) and (3.10) is complicated, but for the special cases $d_{k}=0$ (the limit of nobody disperses, $m \rightarrow 0$, or the limit of no survival in dispersal, $p \rightarrow 0$ ) and $d_{k}=1$ (all disperse, $m=1$ ) we obtain easier expressions.

\subsubsection{Well-mixed metapopulation}

For $d_{k}=1$ the expression (3.7) determining convergence stability becomes

$$
D_{1}^{\prime}\left(s_{\mathrm{res}}\right)=\frac{1}{\langle n F\rangle} \sum_{k=1}^{N} \pi_{k} n_{k}\left(F_{k, \mathrm{SS}}+\left(n_{k}-1\right) F_{k, \mathrm{SD}}\right) .
$$

When $d_{k}=1$, we have $C_{k}=1 / n_{k}^{3}$. Furthermore, all other coefficients in (3.12) are zero, except for $\phi_{\mathrm{SS}}=n_{k}^{3}$, so that

$$
D_{2}\left(s_{\text {res }}\right)=\frac{1}{\langle n F\rangle} \sum_{k=1}^{N} \pi_{k} n_{k} F_{k, \mathrm{SS}} .
$$

The second derivative of metapopulation fitness in the well-mixed situation is thus a biased sum of the second derivatives of the fecundity function with respect to self, which is a rather natural result.

\subsubsection{Limited dispersal}

When $d_{k}=0$, we do not really have metapopulation structure anymore, but it can be analysed as a limit case describing situations with very little dispersal, $m \rightarrow 0$, 
or with very little survival in dispersal, $p \rightarrow 0$. We obtain from (3.7)

$$
D_{1}^{\prime}\left(s_{\text {res }}\right)=\frac{2}{\langle n F\rangle} \sum_{k=1}^{N} \pi_{k} n_{k}^{2}\left(F_{k, \mathrm{SS}}+\left(n_{k}-1\right) F_{k, \mathrm{SD}}\right) .
$$

For $d_{k}=0$ we have $\phi_{k, \mathrm{DD}^{\prime}}=\psi_{k, \mathrm{D} \times \mathrm{D}}=0$. Furthermore, it is convenient to look at the form (3.13), because for $d_{k}=0$ also $\xi_{k, \mathrm{SS}}=0$. The other coefficients used in (3.13) are $C_{k} \frac{\phi_{k, \mathrm{SD}}}{n_{k}-1}=2 n_{k}>0, C_{k} \psi_{k, \mathrm{~S} \times \mathrm{S}}=2 n_{k}\left(2 n_{k}-1\right)>0$ and $C_{k} \psi_{k, \mathrm{~S} \times \mathrm{D}}=$ $-2 n_{k}\left(n_{k}-1\right)<0$. We thus have

$$
\begin{gathered}
D_{2}\left(s_{\mathrm{res}}\right)=\underbrace{\frac{2}{\langle n F\rangle} \sum_{k=1}^{N} \pi_{k} n_{k}^{2}\left(F_{k, \mathrm{SS}}+\left(n_{k}-1\right) F_{k, \mathrm{SD}}\right)}_{=D_{1}^{\prime}\left(s_{\mathrm{res}}\right)} \\
+\frac{2}{\langle n F\rangle} \sum_{k=1}^{N} \pi_{k} n_{k}^{2} F_{k, \mathrm{res}}^{0}\left[\left(2 n_{k}-1\right)\left(\frac{F_{k, \mathrm{~S}}}{F_{k, \mathrm{res}}^{0}}\right)^{2}-\left(n_{k}-1\right)\left(\frac{F_{k, \mathrm{~S}}}{F_{k, \mathrm{res}}^{0}}\right)\left(\frac{F_{k, \mathrm{D}}}{F_{k, \mathrm{res}}^{0}}\right)\right] .
\end{gathered}
$$

For a convergence stable singular strategy $D_{1}^{\prime}\left(s_{\text {res }}\right)<0$ so that the first term in (3.18) is negative. In addition, if the second term is positive and large enough, $D_{2}\left(s_{\text {res }}\right)>0$ and evolutionary branching occurs.

In a homogeneous metapopulation (one patch type only), a strategy is singular when $F_{\mathrm{S}}=0$. In such a situation, the second term in (3.18) is zero, and evolutionary branching is thus impossible for small $d_{k}$, as observed already by Parvinen et al. (2017). The same conclusion applies in such heterogeneous metapopulations, in which the sign of the fecundity derivative $F_{k, \mathrm{~S}}$ does not depend on $k$, but this is not the general situation.

In a heterogeneous metapopulation the derivatives $F_{k, \mathrm{~S}}$ in different patch types are generally not zero, but their biased average (3.5) is. Therefore, some $F_{k, \mathrm{~S}}$

are positive and some negative. Nevertheless, the component $\left(2 n_{k}-1\right)\left(\frac{F_{k, \mathrm{~S}}}{F_{k, \text { res }}^{0}}\right)^{2}$ brings a positive contribution to the second derivative, so we can state that variance in $F_{k, \mathrm{~S}}$ promotes evolutionary branching. From the second term inside the square brackets of (3.18) we can say that a positive correlation in $F_{k, \mathrm{~S}}$ and $F_{k, \mathrm{D}}$ (i.e., $\left.F_{k, \mathrm{~S}} F_{k, \mathrm{D}}>0\right)$ inhibits branching, whereas their negative correlation promotes branching.

\section{Public-goods cooperation}

Suppose that all $n_{k}$ individuals in the same patch are engaged in a single $n_{k}$-person public-goods game. Cost is typically assumed to be a function of the investment level by self, whereas there are two common ways to model the benefit (Sigmund, 2010). 


\begin{tabular}{|c|c|c|}
\hline & Homogeneous model & Heterogeneous model \\
\hline Fitness gradient & Sign-equivalent with $F_{\mathrm{S}}$ & $\begin{array}{l}\text { Biased average of } F_{k, \mathrm{~S}} \text { (equa- } \\
\text { tion 3.5) }\end{array}$ \\
\hline $\begin{array}{l}\text { Second-order derivative } \\
\text { (disruptive selection) }\end{array}$ & $\begin{array}{l}\text { Sign-equivalent with } \\
\phi_{\mathrm{SS}}\left(\frac{F_{\mathrm{SS}}}{F_{\mathrm{res}}^{0}}\right)+\phi_{\mathrm{SD}}\left(\frac{F_{\mathrm{SD}}}{F_{\mathrm{res}}^{0}}\right)+ \\
\phi_{\mathrm{DD}^{\prime}}\left(\frac{F_{\mathrm{DD}}}{F_{\mathrm{res}}^{0}}\right)+\psi_{\mathrm{D} \times \mathrm{D}}\left(\frac{F_{\mathrm{D}}}{F_{\text {res }}^{0}}\right)^{2}\end{array}$ & $\begin{array}{l}\text { Biased average of } \\
\phi_{k, \mathrm{SS}}\left(\frac{F_{k, \mathrm{SS}}}{F_{k, \text { res }}^{0}}\right)+\phi_{k, \mathrm{SD}}\left(\frac{F_{k, \mathrm{SD}}}{F_{k, \text { res }}^{0}}\right)+ \\
\phi_{k, \mathrm{DD}}\left(\frac{F_{k, \mathrm{DD}}}{F_{k, \text { res }}^{\prime}}\right)+\psi_{k, \mathrm{~S} \times \mathrm{S}}\left(\frac{F_{k, \mathrm{~S}}}{F_{k, \text { res }}^{0}}\right)^{2}+ \\
\psi_{k, \mathrm{~S} \times \mathrm{D}}\left(\frac{F_{k, \mathrm{~S}}}{F_{k, \text { res }}^{0}}\right)\left(\frac{F_{k, \mathrm{D}}}{F_{k, \text { res }}^{0}}\right)+\psi_{k, \mathrm{D} \times \mathrm{D}}\left(\frac{F_{k, \mathrm{D}}}{F_{k, \text { res }}^{0}}\right)^{2}\end{array}$ \\
\hline $\begin{array}{l}\text { Evolutionary branching } \\
\text { when } m \approx 0\end{array}$ & Not possible & $\begin{array}{lll}\text { Possible, } & \text { especially } & \text { when } \\
F_{k, \mathrm{~S}} F_{k, \mathrm{D}}<0 & & \end{array}$ \\
\hline $\begin{array}{l}\text { No evolutionary branch- } \\
\text { ing for } m=1 \text {, but evolu- } \\
\text { tionary branching } \\
\text { for some } m<1\end{array}$ & $\begin{array}{l}\text { Not typical. Impossible, if } \\
F_{\mathrm{DD}^{\prime}} \leqslant 0 \text { or } F_{\mathrm{DD}^{\prime}} \leqslant F_{\mathrm{SD}}\end{array}$ & Possible (Figure 2) \\
\hline
\end{tabular}

Table 1: Comparison of the homogeneous and heterogeneous Wright's island model summarising the results from our previous (Parvinen et al., 2017) and current work.

\subsection{Public-goods cooperation without self-benefit}

The benefit of cooperation in a public-goods game can be assumed to be a function of the average investment level of all the other $n_{k}-1$ players in the same patch excluding self, which results in

$$
F_{k}\left(s_{1} ;\left(s_{2}, \ldots, s_{n_{k}}\right)\right)=f_{k}\left(\frac{s_{2}+\cdots+s_{n_{k}}}{n_{k}-1}\right)-g_{k}\left(s_{1}\right),
$$

where $f_{k}$ and $g_{k}$ represent the benefit from public goods and the cost of contribution, respectively. Since fecundity is by definition non-negative, and benefits are obtained only after cost has been paid, we have the requirement $f_{k}(0)-g_{k}(s) \geqslant 0$, which typically results in an upper bound for $s$. The first-order derivatives are given by

$$
F_{k, \mathrm{~S}}=-g_{k}^{\prime}\left(s_{\mathrm{res}}\right), \quad F_{k, \mathrm{D}}=\frac{f_{k}^{\prime}\left(s_{\mathrm{res}}\right)}{n_{k}-1} .
$$

The cost functions $g_{k}$ are typically assumed to be increasing, $g_{k}^{\prime}(s)>0$. Therefore, $F_{k, \mathrm{~S}}<0$ for all $k$. According to Theorem 1, the fitness gradient is then negative, $D_{1}\left(s_{\text {res }}\right)<0$, and the investment level $s$ will always evolve to zero. This generalizes our previous result for a homogeneous metapopulation case (Parvinen et al., 2017).

We observe here that spatial heterogeneity does not affect the evolution of cooperation. Such an observation has been made earlier by Rodrigues and Gardner (2012), see their Figure 1D. However, our next example shows that spatial heterogeneity can have a big impact on the evolution of cooperation. 


\subsection{Public-goods cooperation with self-benefit}

Now the common benefit of cooperation is assumed to be a function of the average investment level of all $n_{k}$ players in the same patch including self, and cooperation is assumed to cause a direct cost to each cooperating individual. In particular, the fecundity function is assumed to be

$$
F_{k}\left(s_{1} ;\left(s_{2}, \ldots, s_{n_{k}}\right)\right)=f_{k}\left(\frac{s_{1}+s_{2}+\cdots+s_{n_{k}}}{n_{k}}\right)-g_{k}\left(s_{1}\right) .
$$

Again, the requirement $f_{k}(0)-g_{k}(s) \geqslant 0$ applies. Both $f_{k}$ and $g_{k}$ are assumed to be increasing functions.

The first-order derivatives are given by

$$
F_{k, \mathrm{~S}}=\frac{f_{k}^{\prime}\left(s_{\mathrm{res}}\right)}{n_{k}}-g_{k}^{\prime}\left(s_{\mathrm{res}}\right), \quad F_{k, \mathrm{D}}=\frac{f_{k}^{\prime}\left(s_{\mathrm{res}}\right)}{n_{k}},
$$

and the second-order derivatives are given by

$$
\begin{aligned}
& F_{k, \mathrm{SS}}=\frac{f_{k}^{\prime \prime}\left(s_{\mathrm{res}}\right)}{n_{k}^{2}}-g_{k}^{\prime \prime}\left(s_{\mathrm{res}}\right), \\
& F_{k, \mathrm{SD}}=F_{k, \mathrm{DD}}=F_{k, \mathrm{DD}^{\prime}}=\frac{f_{k}^{\prime \prime}\left(s_{\mathrm{res}}\right)}{n_{k}^{2}} .
\end{aligned}
$$

Our framework provides analytic expressions for the selection gradient (3.5) and the conditions for convergence stability (3.7) and evolutionary stability (3.10), in which the derivatives above are used. Singular strategies satisfying $D_{1}\left(s^{*}\right)=$ 0 can be solved by using the explicit expression (3.5) for the selection gradient $D_{1}\left(s_{\text {res }}\right)$. We do not obtain an explicit solution for $s^{*}$, and thus we solve $s^{*}$ from $D_{1}\left(s^{*}\right)=0$ numerically. Conditions for convergence stability and evolutionary stability are analytically expressed in terms of $s^{*}$, and we thus obtain the results about monomorphic evolution summarized below.

\subsubsection{Spatial heterogeneity promotes evolutionary branching of coop- eration}

As an illuminating example in which spatial heterogeneity promotes evolutionary branching, we show a concrete result where we have two kinds of patches with sizes $n_{1}$ and $n_{2}$ in proportions $\pi_{1}$ and $\pi_{2}=1-\pi_{1}$. The benefit and cost functions are assumed to be

$$
f_{k}(s)=f_{0}+\frac{\rho_{k} s}{1+\kappa \rho_{k} s}, \quad g_{k}(s)=s .
$$

The condition $f_{k}(0)-g_{k}(s) \geqslant 0$ results in the upper bound $s \leqslant f_{0}$.

The derivative

$$
F_{k, \mathrm{~S}}=\frac{f^{\prime}(s)}{n}-g^{\prime}(s)=\frac{\rho_{k}}{n_{k}\left(1+\kappa \rho_{k} s\right)^{2}}-1
$$


is a decreasing function of $s$, and for $s=0$ we have $F_{k, S}=\rho_{k} / n_{k}-1$. For cooperation to evolve, at least for one $k$ we must have $\rho_{k}>n_{k}$. In the following parameter values are chosen so that positive investment is preferred in patch type $1\left(\rho_{1}>n_{1}\right)$ and zero investment is preferred in patch type $2\left(\rho_{2}<n_{2}\right)$.

Figure 2 interestingly shows that evolutionary branching of cooperation is possible in the heterogeneous metapopulation (intermediate $\pi_{1}$ ). Because the Holling II-type function $f_{k}$ is strictly concave, $f_{k}^{\prime \prime}\left(s_{\mathrm{res}}\right)<0$ and thus $F_{k, \mathrm{SS}}<0$. Therefore for $m=1$ we have $D_{2}\left(s_{\text {res }}\right)<0$ from (3.16). Evolutionary branching does not occur when $m=1$, but decreasing $m$ can result in evolutionary branching. In other words, unlike in the homogeneous metapopulation, spatial structure can promote evolutionary branching. This effect is present both when patch types differ only in their size $n_{1} \neq n_{2}$, and when patch sizes are the same, but the fecundity functions differ $\left(\rho_{1} \neq \rho_{2}\right)$. Meszéna et al. (1997) found a similar result in a two-patch model about patch specialization. In their Figure 6, for intermediate values of patch difference, a generalist ESS becomes a branching point when the migration rate is decreased (inverse migration rate is increased). Also Nurmi and Parvinen (2008) found an analogous result concerning the evolution of resource specialization in a spatially heterogeneous metapopulation model. Their Figure 8b illustrates that for complementary resources, a generalist ESS can turn into a branching point when the emigration probability is decreased.

Parvinen et al. (2017) showed that homogeneous spatial structure does not favor evolutionary branching in this type of public-good cooperation. Their Theorem 5 states for homogeneous models that if a singular strategy $s$ is evolutionarily stable and convergence stable in a well-mixed model $(m=1)$, and $F_{\mathrm{DD}^{\prime}} \leqslant 0$ or $F_{\mathrm{DD}^{\prime}} \leqslant F_{\mathrm{SD}}$, then $s$ is also evolutionarily stable and convergence stable for $0<m<1$. For the current model $F_{\mathrm{DD}^{\prime}}=F_{\mathrm{SD}}$, and thus the conditions of their Theorem 5 are satisfied. As $F_{\mathrm{SS}}<0$ and thus evolutionary branching does not occur in the well-mixed case $(m=1)$, it does not occur for any $m \leqslant 1$ for the homogeneous model. The cases $\pi_{1}=0$ and $\pi_{2}=0$ correspond to homogeneous landscapes, in which the singular strategies and their convergence stability is independent of $m$. For $\pi_{1}=0$ all patches are of type 2 , and since $\rho_{2}<n_{2}$, the strategy not to cooperate is evolutionarily attracting. For $\pi_{1}=1$ all patches are of type 1 , and since $\rho_{1}>n_{1}$, cooperation does evolve. By solving $F_{k, \mathrm{~S}}=0$ from (4.7), we obtain in the homogeneous case

$$
s^{*}=\frac{\sqrt{\rho}-\sqrt{n}}{\rho \kappa \sqrt{n}}>0,
$$

which is a singular, evolutionarily attracting and uninvadable strategy, when $\rho>n$, provided that $s^{*}<f_{0}$, otherwise $s=f_{0}$ is an evolutionarily attracting boundary strategy. For the chosen parameters in Figure 2 the singular strategy given by $(4.8)$ is $s^{*} \approx 0.93$. 
a)

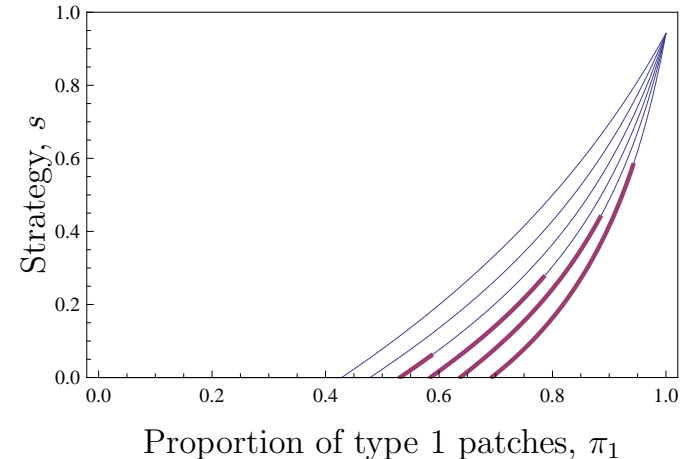

c)

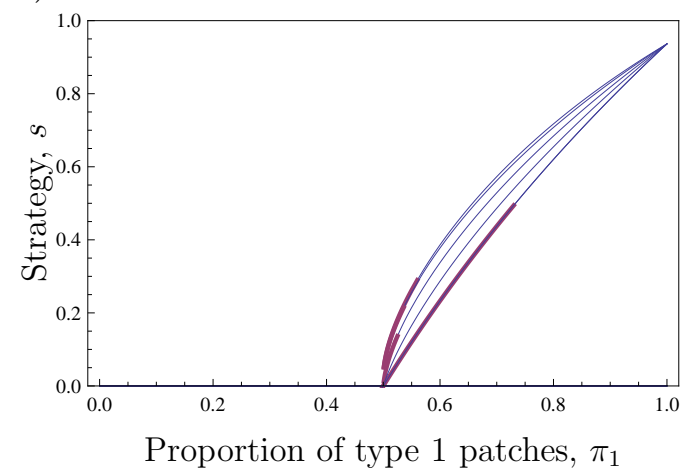

b)

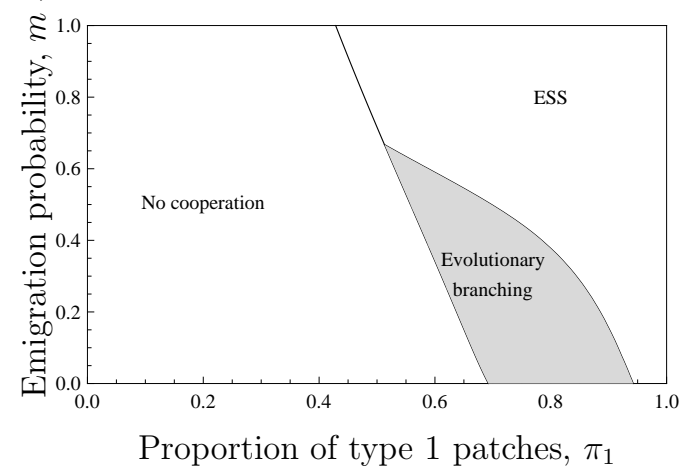

d)

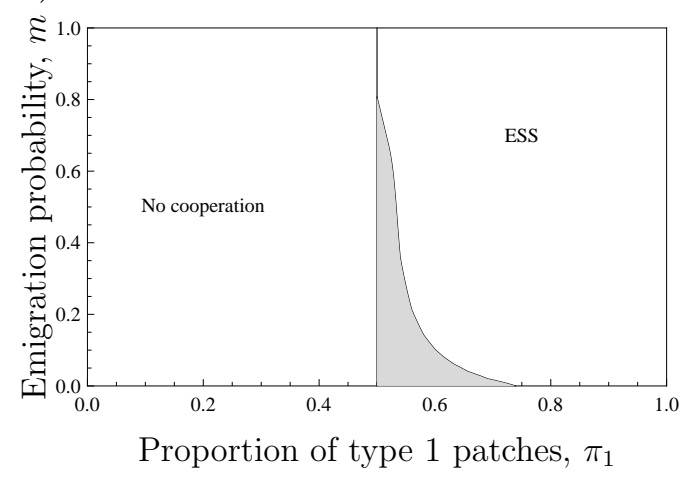

Figure 2: Heterogeneous landscape can favor evolutionary branching of cooperation. (a, c) Singular strategies as a function of $\pi_{1}$ for different values of $m$. Branching points are shown in bold. (b, d) Parameter regions showing the type of the evolutionarily attracting monomorphic strategy. The gray area shows when the singular strategy is a branching point. Parameters: (a, b) $n_{1}=4, n_{2}=9$, $\rho_{1}=\rho_{2}=6(\mathrm{c}, \mathrm{d}) \rho_{1}=6 \rho_{2}=2, n_{1}=n_{2}=4$. Common parameters: $\kappa=0.04$, $p=0.95, f_{0}=1$.

\subsubsection{Evolutionary branching results in the coexistence of cooperators and defectors}

Evolutionary branching means that the strategy of a monomorphic population first evolves near a singular strategy, after which the monomorphic population becomes dimorphic, and the traits of the two morphs evolve away from each other. In order to determine what happens as a result of evolutionary branching, we need to find the equilibria of dimorphic population dynamics, and calculate the invasion fitness of a mutant in the environment set by the dimorphic resident population. Methods for such analysis for the heterogeneous Wright's island model are presented in Appendix A.

Two strategies are mutually invadable, if one strategy has positive invasion fitness when the other strategy is resident and vice versa. Using the metapopulation fitness notation, this means $R_{\mathrm{m}}\left(s_{1}, s_{2}\right)>1$ and $R_{\mathrm{m}}\left(s_{2}, s_{1}\right)>1$. Mutually invadable strategy pairs can be found, e.g., by checking from a pairwise invadability plot 
(PIP) which strategy pairs satisfy the condition above. The left-side parts of each panel in Figure 3 consist of pairwise invadability plots for different values of $\pi_{1}$, the proportion of type 1 patches. The right-side parts illustrate the corresponding sets of mutual invadability, which in this case are equal to the sets of strategy pairs that can coexist. The coexistence of two mutually invadable strategies is called protected (Geritz et al., 1999). Mutual invadability is, however, not a necessary condition for coexistence, and thus the coexistence of two strategies could also be unprotected. By calculating the invasion fitnesses of mutants in an environment set by a dimorphic resident population we can determine the dimorphic fitness gradient, and thus also plot the isoclines of the dimorphic fitness gradient (Figure 3) (Geritz et al., 1999). From such plots we can determine dimorphic strategy coalitions (strategy pairs), that are evolutionarily attracting (convergence stable). Such strategies, together with monomorphic singular strategies, are plotted in Figure 4 as a function of $\pi_{1}$.

For parameters chosen in Figures 3 and 4, we have $\rho_{2}<n_{2}$ and the selfbenefit of cooperation does not exceed costs in patches of type 2. Therefore, when patches of type 2 have strong influence enough $\left(\pi_{1} \lesssim 0.64\right)$, the strategy $s=0$ is evolutionarily attracting, and monomorphic evolution thus results in no cooperation (Figure 4). Nevertheless, defectors $(s=0)$ and full cooperators $\left(s=f_{0}=1\right)$ may coexist in an uninvadable dimorphic strategy coalition, which is locally evolutionarily attracting, but not reachable from an initially monomorphic population (Figure 3a).

When $\pi_{1}$ is increased, a positive evolutionarily attracting singular strategy appears. The singular strategy is evolutionarily attracting, and its cooperation level is an increasing function of $\pi_{1}$. For intermediate values of $\pi_{1}\left(0.64 \lesssim \pi_{1} \lesssim\right.$ 0.88 ) corresponding to significant spatial heterogeneity, this singular strategy is not uninvadable, i.e., it is a branching point. Figure 3bc illustrates that dimorphic evolution then results in the evolutionarily stable coexistence of defectors $(s=0)$ and cooperators $(s>0)$.

When $\pi_{1}$ is increased further, the positive monomorphic singular strategy becomes uninvadable (evolutionarily stable). An initially monomorphic population thus remains monomorphic, and its strategy evolves to the singular strategy. However, a dimorphic strategy coalition can still be locally evolutionarily attracting (Figure 3e). When $\pi_{1}$ is large enough, also an initially dimorphic population becomes monomorphic through evolution (Figure 3fg).

Altogether, Figures 3 and 4 illustrate that for significant spatial heterogeneity, evolutionary branching can result in the evolutionarily stable coexistence of defectors and cooperators.

\section{Discussion}

Unveiling the source of disruptive selection contributes to our understanding of within-species polymorphism (Mather, 1955) as well as a potential mechanism of speciation (Maynard Smith, 1966; Doebeli, 2011; Dieckmann and Doebeli, 1999). 


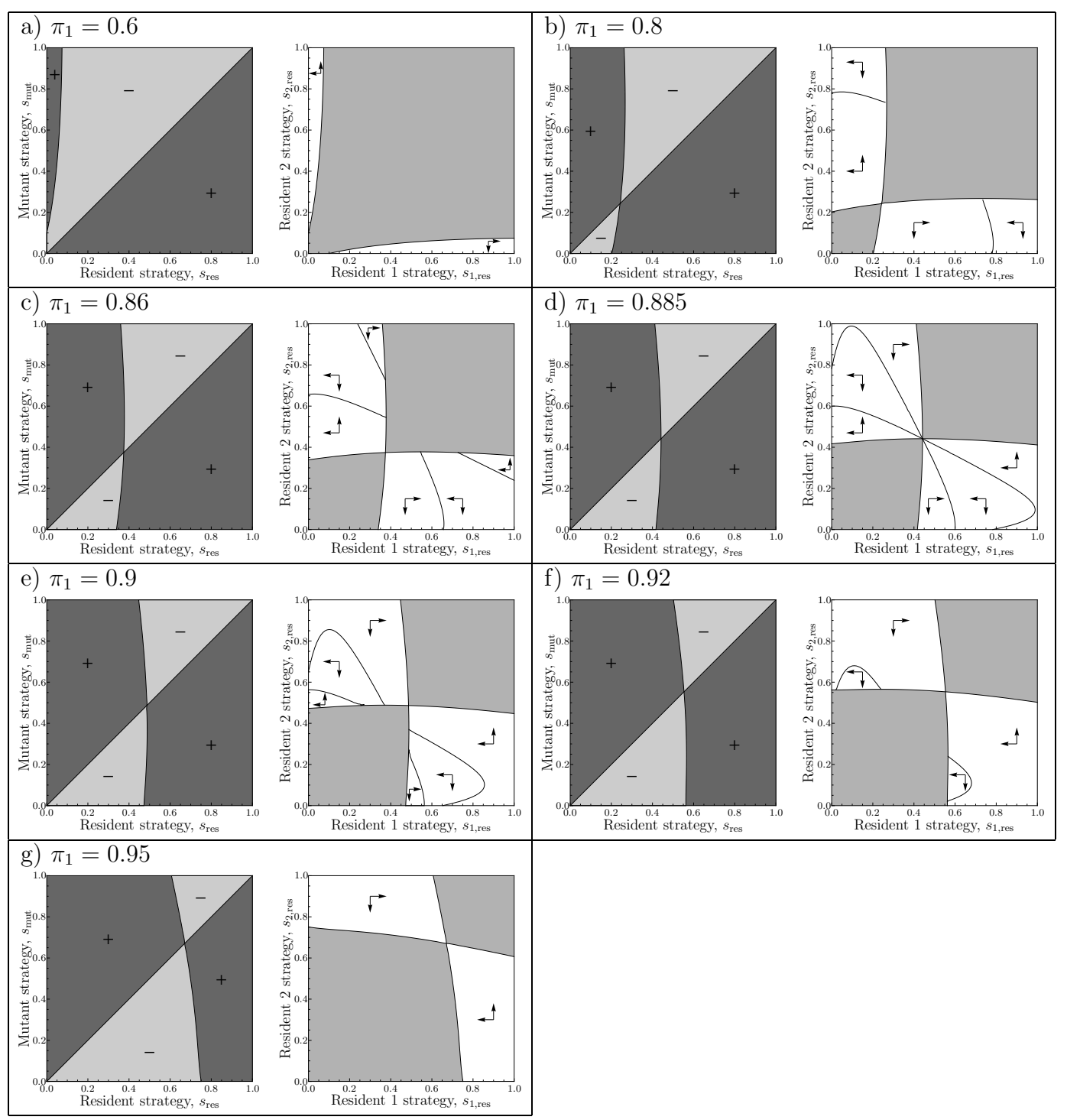

Figure 3: Evolutionary branching can result in the coexistence of cooperators and defectors. The left-side parts show pairwise invadability plots, and the right-side parts show the corresponding sets of (protected) dimorphisms (white areas) with the isoclines of the dimorphic fitness gradient for different values of the proportion of type 1 patches $\pi_{1}$. In the pairwise invadability plots, areas in which a mutant with strategy $s_{\text {mut }}$ has positive fitness (i.e., $R_{\mathrm{m}}\left(s_{\text {mut }}, s_{\text {res }}\right)>1$ ) in the environment set by a resident population with strategy $s_{\text {res }}$ are plotted in dark gray. Light gray corresponds to negative fitness. Parameters: $n_{1}=3, n_{2}=9, \rho_{1}=7, \rho_{2}=6$, $\kappa=0.08, p=0.95, m=0.3, f_{0}=1$. 


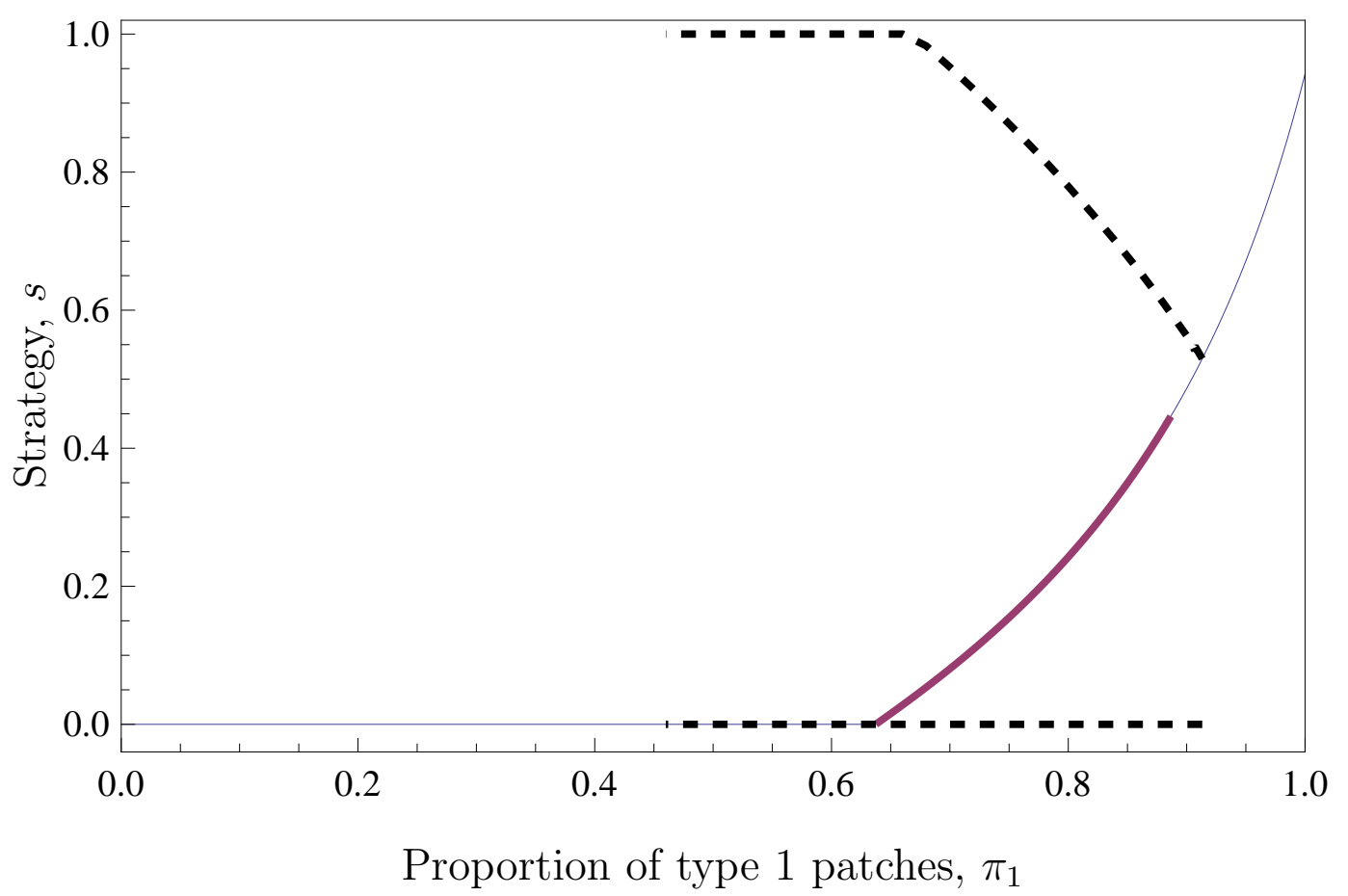

Figure 4: Strategies in the evolutionarily singular dimorphic strategy coalition (dashed curves) and monomorphic singular strategies (solid curve) as a function of $\pi_{1}$. Monomorphic branching points are shown in bold. Parameters as in Figure 3.

In this paper we have studied the effect of spatially heterogeneous population structure on the force of disruptive selection, by calculating metapopulation fitness of mutants and investigating its first- and second-order derivatives. To this end, we have adopted a spatially heterogeneous Wright's island model and studied the evolution of a fecundity-affecting trait, $s$. The present paper is a sequel to Parvinen et al. (2017), which described conditions for evolutionary branching in a spatially homogeneous Wright's island model in terms of fecundity derivatives. A comparison between these two models, therefore, elucidates essential roles that spatial heterogeneity plays in evolutionary dynamics. In Appendix B, we have performed a separate analysis on the same model by employing relatedness-based arguments in order to clarify the connection to previous literature, and confirmed that the first-order effect of selection has a clear interpretation as an inclusive fitness that contains relevant reproductive values.

Our spatially heterogeneous model included different patch types, labeled by $k$, and we assumed that the frequency of type- $k$ patches among all patches is given by $\pi_{k}$ and that the relative productivity (called reproductive potential) of a type- $k$ patch, given all individuals adopt a resident strategy, is given by $V_{k}$ (see Eq. (3.2) for its precise definition). Local population sizes, $n_{k}$, are finite and may differ for different patch types. Under these settings, we have found that the first-order derivative of the metapopulation fitness of mutants in a heterogeneous 
population is a weighted average of the corresponding derivative in a homogeneous population, where the corresponding term for patch-type $k$ should be weighted by $\pi_{k} V_{k}$ (see Theorem 1). We have also found that the same principle applies to the second-order derivative (see Theorem 3).

An investigation of the second-order derivative of metapopulation fitness in Section 3.4 revealed that for limited dispersal the variance of $F_{k, \mathrm{~S}}$ over different patch types $k$ positively contributes to evolutionary branching. Note that the weighted average of $F_{k, \mathrm{~S}}$ is equal to zero at an evolutionarily singular point, $s^{*}$, and therefore some of $F_{k, \mathrm{~S}}$ 's should be positive and the others should be negative unless all of them are null. In others words, large variance in $F_{k, \mathrm{~S}}$ means that in some patches having a larger trait $s$ is very beneficial in terms of fecundity for the bearer, whereas it is very harmful in other patches. It is quite natural that under such heterogeneous environments evolutionary diversification of traits should occur. Therefore our result theoretically confirms our intuitive prediction. Our result is in line with Doebeli and Dieckmann (2003) in that spatial heterogeneity strengthens the degree of local adaptation, and thus it increases disruptive selection.

Another condition that we found for evolutionary branching was that a negative correlation between $F_{k, \mathrm{~S}}$ and $F_{k, \mathrm{D}}$, namely $F_{k, \mathrm{~S}} F_{k, \mathrm{D}}<0$ favors evolutionary branching. This occurs in two cases. In the first case, $F_{k, \mathrm{~S}}<0$ and $F_{k, \mathrm{D}}>0$, suggesting that a larger trait is costly to the bearer but beneficial to others, both in terms of their fecundities. In the second case, $F_{k, \mathrm{~S}}>0$ and $F_{k, \mathrm{D}}<0$, suggesting that a larger trait is beneficial to the bearer but costly to others, both in terms of their fecundities. In sum, evolutionary branching is favored if the effects of one's trait on one's fecundity and on others' fecundities have the opposite signs, which is a novel result that the current paper elucidated.

As an application we studied a simple model of public-goods cooperation and examined the possibility of evolutionary branching to two morphs, namely cooperators and defectors. We have previously shown that in a homogeneously spatially structured population (for a wide class of fecundity functions, including publicgoods cooperation), if evolutionary branching does not occur in a randomly mixing condition of $m=1$ (where $m$ is the migration rate), then for any $0<m<1$ evolutionary branching does not occur, either (Parvinen et al., 2017). Our general conclusion there was that spatiality generally disfavors evolutionary branching unless some special condition is met (Parvinen et al., 2017). However, spatial heterogeneity breaks this principle, and in fact we found in the present paper that evolutionary branching does occur in a heterogeneously spatially structured population at small migration rates $m$, even if evolutionary branching does not occur in a corresponding well-mixed case $(m=1)$. This result clearly suggests that spatial heterogeneity is one of the factors that drive evolutionary diversification (Doebeli and Dieckmann, 2003; Heinz et al., 2009; Payne et al., 2011; Haller et al., 2013).

In this paper, we have concentrated on the evolution and evolutionary branching of fecundity-affecting traits, $s$. Traits that affect other life-history parameters than fecundity, such as mortality, migration rate, survival rate in migration, patch 
extinction rates, are also important, and revealing corresponding conditions for evolutionary branching in terms of those life-history parameters and their derivatives would be an important study. For such an attempt for a homogeneously structured population under a weak selection approximation, we refer to Mullon et al. $(2016,2018)$. An extension to a heterogeneously spatially structured population would give us more insights about the role that heterogeneity plays in evolutionary branching of traits. We have studied spatial heterogeneity only, but another conceivable dimension of heterogeneity is temporal heterogeneity. It is interesting to theoretically reveal how temporal heterogeneity or spatio-temporal heterogeneity (Rodrigues and Gardner, 2012) of habitats affects evolutionary stability and branching conditions of an interesting trait.

\section{Acknowledgements}

The authors wish to thank SOKENDAI (the Program to Invite Foreign Researchers at SOKENDAI) for hosting K.P. for two months in Spring 2017, during which the majority of the presented research was conducted. This work is supported by JSPS KAKENHI Grant Numbers 15H04425 and 16H06324 to H.O., and Grant Numbers 16K05283 and 16H06412 to J.Y.W. We also want to thank anonymous reviewers for their comments.

\section{A Dimorphic population}

\section{A.1 Resident population}

Consider a dimorphic population, i.e., a population with two residents with strategies $\left(s_{1}, m_{1}\right)$ and $\left(s_{2}, m_{2}\right)$. In each patch type, let $b_{k, i}$ denote the probability that in a patch of type $k$, there are $i$ resident 1 adults, and $n_{k}-i$ resident 2 adults. The amounts of juveniles in such a patch before dispersal are

$$
\begin{aligned}
& x_{k, i}=i F\left(s_{1} ;(\underbrace{s_{1}, \ldots, s_{1}}_{i-1}, \underbrace{s_{2}, \ldots, s_{2}}_{n_{k}-i})\right) \\
& y_{k, i}=\left(n_{k}-i\right) F\left(s_{2} ;(\underbrace{s_{1}, \ldots, s_{1}}_{i}, \underbrace{s_{2}, \ldots, s_{2}}_{n_{k}-i-1})\right) .
\end{aligned}
$$

The amount of immigrants of each type are

$$
\begin{aligned}
& I_{1}=\sum_{k=1}^{N} \pi_{k} \sum_{i=1}^{n_{k}} b_{k, i} m_{1} x_{k, i} \\
& I_{2}=\sum_{k=1}^{N} \pi_{k} \sum_{i=1}^{n_{k}} b_{k, i} m_{2} y_{k, i} .
\end{aligned}
$$

As a result, the proportion of resident 1 juveniles in a patch with $i$ resident 1 adults, and $n_{k}-i$ resident 2 adults is 


$$
\hat{p}_{k, i}=\frac{\left(1-m_{1}\right) x_{k, i}+I_{1}}{\left(1-m_{1}\right) x_{k, i}+I_{1}+\left(1-m_{1}\right) y_{k, i}+I_{2}} .
$$

The probability that a patch with $i$ residents of type 1 will have $j$ residents of type 1 in the next year is binomial

$$
\hat{t}_{k, j i}=\left(\begin{array}{c}
n_{k} \\
j
\end{array}\right)\left(\hat{p}_{k, i}\right)^{j}\left(1-\hat{p}_{k, i}\right)^{n_{k}-j}
$$

We collect these values into the transition matrix $\hat{\mathbf{T}}_{k}=\left(\hat{t}_{k, j i}\right)$. Let $B_{k}=\left(b_{k, 0}, b_{k, 1}, \ldots, b_{k, n_{k}}\right)$ denote the probability distribution vector in patches of type $k$. We thus obtain

$$
B_{k}(t+1)=\hat{T}_{k}(B(t)) B_{k}(t)
$$

where the notation $\hat{T}_{k}(B(t))$ underscores the fact that the transition matrix $\hat{T}_{k}$ at time $t$ depends on the probability distributions of all patch types through the amounts of immigrants $I_{1}$ and $I_{2}$. By applying (A.5) we can obtain the probability distribution of resident populations at equilibrium.

\section{A.2 Fitness of a mutant}

\section{A.2.1 Initial distribution $\alpha^{0}$}

A dispersing mutant juvenile arrives with probability $\pi_{k}$ into a patch of type $k$, and with probability $b_{k, i}$ that patch has $i$ residents of type $i$ and $n_{k}-i$ residents of type 2. The mutant will compete with $\gamma\left(\left(1-m_{1}\right) x_{k, i}+I_{1}+\left(1-m_{1}\right) y_{k, i}+I_{2}\right)$ resident juveniles, so that for large $\gamma$

$$
\tilde{P}_{k, i}=\gamma P_{k, i}=\frac{n_{k}}{\left(1-m_{1}\right) x_{k, i}+I_{1}+\left(1-m_{1}\right) y_{k, i}+I_{2}} .
$$

Since the mutant is rare, the other $n_{k}$ adults will be residents. Their distribution is given by the binomial distribution. When we sum over the different combinations of adult residents, we get the probability for the number of adults in the next year:

$$
\alpha_{k, j, n_{k}-j-1}^{0}=\sum_{i=0}^{n_{k}} b_{k, i} \tilde{P}_{k, i}\left(\begin{array}{c}
n_{k}-1 \\
j
\end{array}\right)\left(\hat{p}_{k, i}\right)^{j}\left(1-\hat{p}_{k, i}\right)^{n_{k}-j-1}
$$

and $\alpha_{k, j, l}^{0}=0$ for $j+l<n_{k}-1$.

\section{A.2.2 Transition matrix}

Consider now a patch with adult numbers $i, j$ and $n_{k}-i-j$ corresponding to residents of type 1 , residents of type 2 , and mutants, respectively. The amounts of juveniles they get are 


$$
\begin{aligned}
& x_{k, i, j}=i F\left(s_{1} ;(\underbrace{s_{1}, \ldots, s 1}_{i-1}, \underbrace{s_{2}, \ldots, s_{2}}_{j}, \underbrace{s_{\mathrm{mut}}, \ldots, s_{\mathrm{mut}}}_{n_{k}-i-j})\right) \\
& y_{k, i, j}=j F\left(s_{2} ;(\underbrace{s_{2}, \ldots, s 1}_{i}, \underbrace{s_{2}, \ldots, s_{2}}_{j-1}, \underbrace{s_{\mathrm{mut}}, \ldots, s_{\mathrm{mut}}}_{n_{k}-i-j})\right) \\
& z_{k, i, j}=\left(n_{k}-i-j\right) F\left(s_{\mathrm{mut}} ;(\underbrace{s_{1}, \ldots, s 1}_{i}, \underbrace{s_{2}, \ldots, s_{2}}_{j}, \underbrace{s_{\mathrm{mut}}, \ldots, s_{\mathrm{mut}}}_{n_{k}-i-j-1})\right) .
\end{aligned}
$$

The proportion of resident 1, 2 and mutant juveniles after dispersal are

$$
(\underbrace{\frac{\left(1-m_{1}\right) x_{k, i, j}+I_{1}}{W_{k, i, j}}}_{=a_{k, i, j}}, \underbrace{\frac{\left(1-m_{2}\right) y_{k, i, j}+I_{2}}{W_{k, i, j}}}_{=b_{k, i, j}}, \underbrace{\frac{\left.\left(1-m_{\mathrm{mut}}\right) z_{k, i, j}\right)}{W_{k, i, j}}}_{=c_{k, i, j}}),
$$

where $W_{k, i, j}=\left(1-m_{1}\right) x_{k, i, j}+I_{1}+\left(1-m_{2}\right) y_{k, i, j}+I_{2}+\left(1-m_{\text {mut }}\right) z_{k, i, j}$. The transition probabilities are

$$
\operatorname{Pr}\left((i, j) \rightarrow\left(i^{\prime}, j^{\prime}\right)\right)=\frac{n_{k} !}{i^{\prime} ! j^{\prime} !\left(n_{k}-i^{\prime}-j^{\prime}\right) !}\left(a_{k, i, j}\right)^{i^{\prime}}\left(b_{k, i, j}\right)^{j^{\prime}}\left(c_{k, i, j}\right)^{n_{k}-i^{\prime}-j^{\prime}} .
$$

We collect these probabilities into a matrix, and then proceed as in the monomorphic resident case.

\section{B First-order results in terms of inclusive-fitness}

\section{B.1 Fitness}

We regard adults in patches of different types as individuals in different "classes" (Taylor, 1990). Specifically, we call an adult in a type- $k$ patch a "class $k$ individual" in the following. Let us calculate the class- $l$ fitness of a focal class- $k$ individual, denoted by $w_{l, k}$. Suppose that the trait of the focal individual is $s_{1}$ and that the traits of the other $n_{k}-1$ individuals in the same patch are $\boldsymbol{s}_{n_{k}-1}=\left(s_{2}, \cdots, s_{n_{k}}\right)$. Since we assume that there are infinitely many patches, that mutants are rare, and that genetic association between individuals from different patches can be neglected, we can calculate mutant fitness by assuming that the others in different patches adopt the resident trait, $s_{\text {res }}$. Hence we have

$$
\begin{aligned}
& w_{l, k}\left(s_{1}, \boldsymbol{s}_{n_{k}-1}\right)=\underbrace{\delta_{k, l} n_{k} \frac{(1-m) F_{k}\left(s_{1} ; \boldsymbol{s}_{n_{k}-1}\right)}{(1-m) n_{k} \bar{F}_{k}\left(s_{1}, \boldsymbol{s}_{n_{k}-1}\right)+I_{\text {res }}}}_{\text {philopatric component }} \\
& +\underbrace{n_{l} \frac{p \pi_{l} m F_{k}\left(s_{1} ; \boldsymbol{s}_{n_{k}-1}\right)}{(1-m) n_{l} \bar{F}_{l}\left(s_{\text {res }}, s_{\text {res }}, \cdots, s_{\text {res }}\right)+I_{\text {res }}}}_{\text {allopatric component }}
\end{aligned}
$$


where the symbol $\delta_{k, l}$ is the Kronecker delta defined as

$$
\delta_{k, l}=\left\{\begin{array}{ll}
1 & (k=l) \\
0 & (k \neq l)
\end{array},\right.
$$

$\bar{F}_{k}$ represents the average fecundity in a patch of type $k$ where individuals use $\left(s_{1}, \cdots, s_{n_{k}}\right)$, and $I_{\text {res }}$ represents the immigration force;

$$
I_{\text {res }}=p m \sum_{l^{\prime}=1}^{N} \pi_{l^{\prime}} n_{l^{\prime}} \bar{F}_{l^{\prime}}\left(s_{\mathrm{res}}, \cdots, s_{\mathrm{res}}\right) .
$$

We apply the method of Taylor and Frank (1996) here; we think of $s_{2}, \cdots, s_{n_{k}}$ as if they were functions of $s_{1}$, and take the derivative of $w_{l, k}$ with respect to $s_{1}$. We have

$$
\begin{aligned}
\frac{\mathrm{d} w_{l, k}}{\mathrm{~d} s_{1}} & =\sum_{i=1}^{n_{k}} \frac{\partial w_{l, k}}{\partial s_{i}} \frac{\mathrm{d} s_{i}}{\mathrm{~d} s_{1}} \\
& =\frac{\partial w_{l, k}}{\partial s_{1}} \frac{\mathrm{d} s_{1}}{\mathrm{~d} s_{1}}+\left(n_{k}-1\right) \frac{\partial w_{l, k}}{\partial s_{2}} \frac{\mathrm{d} s_{2}}{\mathrm{~d} s_{1}} .
\end{aligned}
$$

At the last equal sign we took advantage of the symmetry between $s_{2}, \cdots, s_{n_{k}}$ in the fitness function, Eq.(B.1). Note that later we will replace $\mathrm{d} s_{1} / \mathrm{d} s_{1}$ with the relatedness to self, 1 , and replace $\mathrm{d} s_{2} / \mathrm{d} s_{1}$ with the relatedness between two different adults in the same type- $k$ patch, denoted by $R_{k}$. All derivatives of $w_{l, k}$ are evaluated at $s_{1}=\cdots=s_{n_{k}}=s_{\text {res }}$.

Equation (B.4) is further calculated in terms of derivatives of $F_{k}$ and $\bar{F}_{k}$ by using the chain rule;

$$
\frac{\mathrm{d} w_{l, k}}{\mathrm{~d} s_{1}}=\left(\frac{\partial w_{l, k}}{\partial F_{k}} \frac{\partial F_{k}}{\partial s_{1}}+\frac{\partial w_{l, k}}{\partial \bar{F}_{k}} \frac{\partial \bar{F}_{k}}{\partial s_{1}}\right) \frac{\mathrm{d} s_{1}}{\mathrm{~d} s_{1}}+\left(n_{k}-1\right)\left(\frac{\partial w_{l, k}}{\partial F_{k}} \frac{\partial F_{k}}{\partial s_{2}}+\frac{\partial w_{l, k}}{\partial \bar{F}_{k}} \frac{\partial \bar{F}_{k}}{\partial s_{2}}\right) \frac{\mathrm{d} s_{2}}{\mathrm{~d} s_{1}},
$$

where all derivatives of $w_{l, k}$ are evaluated at $F_{k}=\bar{F}_{k}=F_{k \text {,res }}^{0}$, and all derivatives of $F_{k}$ and $\bar{F}_{k}$ are evaluated at $s_{1}=\cdots=s_{n_{k}}=s_{\text {res }}$. It is straightforward to calculate these derivatives, as

$$
\left\{\begin{aligned}
\frac{\partial w_{l, k}}{\partial F_{k}} & =\delta_{k, l} n_{k} \frac{(1-m)}{(1-m) n_{k} F_{k, \text { res }}^{0}+I_{\text {res }}}+n_{l} \frac{p \pi_{l} m}{(1-m) n_{l} F_{l, \mathrm{res}}^{0}+I_{\text {res }}} \\
\frac{\partial w_{l, k}}{\partial \bar{F}_{k}} & =-\delta_{k, l} n_{k}^{2} \frac{(1-m)^{2} F_{k, \text { res }}^{0}}{\left\{(1-m) n_{k} F_{k, \text { res }}^{0}+I_{\text {res }}\right\}^{2}} \\
\frac{\partial F_{k}}{\partial s_{1}} & =F_{k, \mathrm{~S}} \\
\frac{\partial F_{k}}{\partial s_{2}} & =F_{k, \mathrm{D}} \\
\frac{\partial \bar{F}_{k}}{\partial s_{i}} & =\frac{F_{k, \mathrm{~S}}+\left(n_{k}-1\right) F_{k, \mathrm{D}}}{n_{k}} \quad(i=1,2)
\end{aligned}\right.
$$




\section{B.2 Relatedness}

Let

$$
d_{k}=\frac{I_{\mathrm{res}}}{(1-m) n_{k} F_{k, \mathrm{res}}^{0}+I_{\mathrm{res}}}
$$

be the backward migration probability from a type- $k$ patch. Then the relatedness between two different adults in the same type- $k$ patch satisfies the recursion

$$
R_{k}=\left(1-d_{k}\right)^{2} \underbrace{\left[\frac{1}{n_{k}}+\left(1-\frac{1}{n_{k}}\right) R_{k}\right]}_{\equiv R_{k}^{\mathrm{R}}}
$$

which has the following solution;

$$
R_{k}=\frac{\left(1-d_{k}\right)^{2}}{n_{k}-\left(n_{k}-1\right)\left(1-d_{k}\right)^{2}} .
$$

\section{B.3 Reproductive value}

Let $v_{k}$ be the individual reproductive value of a class $k$ individual. It is known that the row vector $\boldsymbol{v}=\left(v_{1}, \cdots, v_{N}\right)$ is the left eigenvector of the matrix $\boldsymbol{W}^{(0)}=\left\{w_{l, k}^{(0)}\right\}$, where $w_{l, k}^{(0)}$ is the value of $w_{l, k}$ evaluated for the monomorphic population of $s_{\text {res }}$. A calculation shows

$$
\begin{aligned}
w_{l, k}^{(0)} & =\delta_{k, l} n_{k} \frac{(1-m) F_{k, \mathrm{res}}^{0}}{(1-m) n_{k} F_{k, \mathrm{res}}^{0}+I_{\mathrm{res}}}+n_{l} \frac{p \pi_{l} m F_{k, \mathrm{res}}^{0}}{(1-m) n_{l} F_{l, \mathrm{res}}^{0}+I_{\mathrm{res}}} \\
& =\delta_{k, l} \underbrace{\frac{(1-m) n_{k} F_{k, \mathrm{res}}^{0}}{(1-m) n_{k} F_{k, \mathrm{res}}^{0}+I_{\mathrm{res}}}}_{=1-d_{k}}+\frac{F_{k, \mathrm{res}}^{0}}{F_{l, \mathrm{res}}^{0}} \underbrace{\frac{I_{\mathrm{res}}}{(1-m) n_{l} F_{l, \mathrm{res}}^{0}+I_{\mathrm{res}}}}_{=d_{l}} \cdot \frac{p m \pi_{l} n_{l} F_{l, \mathrm{res}}^{0}}{I_{\mathrm{res}}} \\
& =\delta_{k, l}\left(1-d_{k}\right)+\frac{d_{l}}{F_{l, \mathrm{res}}^{0}} F_{k, \mathrm{res}}^{0} \cdot \frac{p m \pi_{l} n_{l} F_{l, \mathrm{res}}^{0}}{I_{\mathrm{res}}} .
\end{aligned}
$$

Here we claim that $v_{k}=F_{k, \text { res }}^{0} / d_{k}$ gives an (unnormalized) left eigenvector of $\boldsymbol{W}^{(0)}$. In fact we have

$$
\sum_{l=1}^{N} \frac{F_{l, \text { res }}^{0}}{d_{l}} w_{l, k}^{(0)}=\frac{F_{k, \text { res }}^{0}}{d_{k}}\left(1-d_{k}\right)+F_{k, \text { res }}^{0} \underbrace{\frac{\sum_{l=1}^{N} p m \pi_{l} n_{l} F_{l, \text { res }}^{0}}{I_{\text {res }}}}_{=1}=\frac{F_{k, \text { res }}^{0}}{d_{k}},
$$

by which the proof is completed. Let $u_{k}$ be the proportion of class $k$ individuals in the population, which is given by

$$
u_{k}=\frac{\pi_{k} n_{k}}{\sum_{l=1}^{N} \pi_{l} n_{l}}=\frac{\pi_{k} n_{k}}{\langle n\rangle}
$$


where the angle brackets represents $\pi$-weighted average of a quantity;

$$
\langle n\rangle \equiv \sum_{l=1}^{N} \pi_{l} n_{l}
$$

We normalize the reproductive value so that $\sum_{k=1}^{N} u_{k} v_{k}=1$ holds, which leads to the following normalized individual reproductive value;

$$
v_{k}=\frac{\langle n\rangle}{\left\langle\frac{n F}{d}\right\rangle} \frac{F_{k, \text { res }}^{0}}{d_{k}}
$$

where

$$
\left\langle\frac{n F}{d}\right\rangle \equiv \sum_{l=1}^{N} \pi_{l} \frac{n_{l} F_{l, \mathrm{res}}^{0}}{d_{l}}
$$

Hereafter we will employ this normalization.

\section{B.4 Inclusive-fitness interpretation}

Define

$$
w_{k} \equiv \sum_{l=1}^{N} v_{l} w_{l, k}
$$

which is the (reproductive-value-weighted) fitness of a class- $k$ individual. Multiplying eq.(B.5) by $v_{l}$ and summing it over $l$ gives us

$$
\begin{aligned}
\frac{\mathrm{d} w_{k}}{\mathrm{~d} s_{1}}= & {\left[\left(\sum_{l=1}^{N} v_{l} \frac{\partial w_{l, k}}{\partial F_{k}}\right) \frac{\partial F_{k}}{\partial s_{1}}+\left(\sum_{l=1}^{N} v_{l} \frac{\partial w_{l, k}}{\partial \bar{F}_{k}}\right) \frac{\partial \bar{F}_{k}}{\partial s_{1}}\right] \frac{\mathrm{d} s_{1}}{\mathrm{~d} s_{1}} } \\
& +\left(n_{k}-1\right)\left[\left(\sum_{l=1}^{N} v_{l} \frac{\partial w_{l, k}}{\partial F_{k}}\right) \frac{\partial F_{k}}{\partial s_{2}}+\left(\sum_{l=1}^{N} v_{l} \frac{\partial w_{l, k}}{\partial \bar{F}_{k}}\right) \frac{\partial \bar{F}_{k}}{\partial s_{2}}\right] \frac{\mathrm{d} s_{2}}{\mathrm{~d} s_{1}},
\end{aligned}
$$

Using $v_{k}=\sum_{l=1}^{N} v_{l} w_{l, k}^{(0)}$ and $\partial w_{l, k} / \partial F_{k}=w_{l, k}^{(0)} / F_{k, \text { res }}^{0}$, it is easy to confirm

$$
\sum_{l=1}^{N} v_{l} \frac{\partial w_{l, k}}{\partial F_{k}}=\frac{v_{k}}{F_{k, \text { res }}^{0}}
$$

It is also easy to confirm

$$
\sum_{l=1}^{N} v_{l} \frac{\partial w_{l, k}}{\partial \bar{F}_{k}}=-v_{k} \frac{(1-m)^{2} n_{k}^{2} F_{k, \text { res }}^{0}}{\left\{(1-m) n_{k} F_{k, \text { res }}^{0}+I_{\text {res }}\right\}^{2}}=-\frac{v_{k}}{F_{k, \text { res }}^{0}}\left(1-d_{k}\right)^{2} .
$$

Therefore we arrive at

$$
\begin{aligned}
\frac{\mathrm{d} w_{k}}{\mathrm{~d} s_{1}}= & {\left[v_{k} \frac{F_{k, \mathrm{~S}}}{F_{k, \text { res }}^{0}}-v_{k}\left(1-d_{k}\right)^{2} \frac{\frac{F_{k, \mathrm{~S}}}{F_{k, \text { res }}^{0}}+\left(n_{k}-1\right) \frac{F_{k, \mathrm{D}}}{F_{k, \text { res }}^{0}}}{n_{k}}\right] \frac{\mathrm{d} s_{1}}{\mathrm{~d} s_{1}} } \\
& +\left(n_{k}-1\right)\left[v_{k} \frac{F_{k, \mathrm{D}}}{F_{k, \text { res }}^{0}}-v_{k}\left(1-d_{k}\right)^{2} \frac{\frac{F_{k, \mathrm{~S}}}{F_{k, \text { res }}^{0}}+\left(n_{k}-1\right) \frac{F_{k, \mathrm{D}}}{F_{k, \text { res }}^{0}}}{n_{k}}\right] \frac{\mathrm{d} s_{2}}{\mathrm{~d} s_{1}} .
\end{aligned}
$$


According to the method of Taylor and Frank (1996), we replace $\mathrm{d} s_{1} / \mathrm{d} s_{1}$ with the relatedness to self, 1 , and replace $\mathrm{d} s_{i} / \mathrm{d} s_{1}\left(2 \leq i \leq n_{k}\right)$ with the relatedness between two different adults in the same type- $k$ patch, $R_{k}$, and obtain the component of inclusive fitness effect through patch- $k$ actors;

$$
\begin{aligned}
& S_{k} \equiv \underbrace{\left[v_{k} \frac{F_{k, \mathrm{~S}}}{F_{k, \mathrm{res}}^{0}}-v_{k}\left(1-d_{k}\right)^{2} \frac{\frac{F_{k, \mathrm{~S}}}{F_{k, \mathrm{res}}^{0}}+\left(n_{k}-1\right) \frac{F_{k, \mathrm{D}}}{F_{k, \mathrm{res}}^{0}}}{n_{k}}\right]}_{=-C_{k}} \cdot 1 \\
& +\left(n_{k}-1\right) \underbrace{\left[v_{k} \frac{F_{k, \mathrm{D}}}{F_{k, \mathrm{res}}^{0}}-v_{k}\left(1-d_{k}\right)^{2} \frac{\frac{F_{k, \mathrm{~S}}}{F_{k, \mathrm{res}}^{0}}+\left(n_{k}-1\right) \frac{F_{k, \mathrm{D}}}{F_{k, \mathrm{res}}^{0}}}{n_{k}}\right]}_{=B_{k}} \cdot R_{k} \\
& =-C_{k}+\left(n_{k}-1\right) B_{k} R_{k},
\end{aligned}
$$

which is a Hamilton's rule, where $C_{k}$ is the fitness cost of adopting a higher trait value, and $B_{k}$ is its fitness benefit enjoyed by a patch-mate of a focal individual, to whom the focal individual is related by $R_{k}$, and there are $\left(n_{k}-1\right)$ such patch-mates.

Certainly there is a correspondence between our eq.(B.21) and eq.(12) in Wakano and Lehmann (2014); our $-C_{k}$ corresponds to their $w_{\mathrm{S}}$ and our $\left(n_{k}-1\right) B_{k}$ corresponds to their $w_{\mathrm{D}}$, though we consider a class-structured population here whereas Wakano and Lehmann (2014) did not.

To distinguish fitness cost/benefit from fecundity cost/benefit, we define

$$
\left\{\begin{aligned}
-\gamma_{k} & \equiv \frac{F_{k, \mathrm{~S}}}{F_{k, \mathrm{res}}^{0}} \\
\beta_{k} & \equiv \frac{F_{k, \mathrm{D}}}{F_{k, \mathrm{res}}^{0}}
\end{aligned}\right.
$$

and call $\gamma_{k}$ (normalized) fecundity cost and call $\beta_{k}$ (normalized) fecundity benefit. Then the patch- $k$ actor component of inclusive fitness effect is rewritten as

$$
S_{k}=v_{k}\left[-\gamma_{k}+\left(n_{k}-1\right) \beta_{k} R_{k}-\left(1-d_{k}\right)^{2}\left\{-\gamma_{k}+\left(n_{k}-1\right) \beta_{k}\right\} R_{k}^{\mathrm{R}}\right],
$$

which has a clearer inclusive fitness interpretation; my use of a deviant strategy causes the decrease of my offspring by $\gamma_{k}$ but it gives the excess of $\beta_{k}$ offspring to each of my $\left(n_{k}-1\right)$ patchmates, to whom I am related by $R_{k}$. However, this causes the total excess of $\left\{-\gamma_{k}+\left(n_{k}-1\right) \beta_{k}\right\}$ offspring born in this patch. With probability $\left(1-d_{k}\right)$ they will compete on their natal patch, and displace random individuals who are native to that patch with probability $\left(1-d_{k}\right)$, to whom the focal individual is related by $R_{k}^{\mathrm{R}}$. Here $R_{k}^{\mathrm{R}}$ is the relatedness of a focal adult to a random adult individual (including self) in the same patch. Therefore the cost of local kin competition amounts to $\left(1-d_{k}\right)^{2}\left\{-\gamma_{k}+\left(n_{k}-1\right) \beta_{k}\right\} R_{k}^{\mathrm{R}}$.

By the relation $R_{k}=\left(1-d_{k}\right)^{2} R_{k}^{\mathrm{R}}$, the expression above is simplified to

$$
S_{k}=v_{k}\left(-\gamma_{k}\right)\left(1-R_{k}\right) \text {. }
$$


It does not contain $\beta_{k}$, which is called Taylor's cancellation result (Taylor 1992) and is specific to the Wright-Fisher demography assumed here (Lehmann and Rousset, 2010). The total inclusive fitness effect, $S_{\mathrm{IF}}$, the sign of which predicts the direction of selection acting on a mutant gene, is the sum of $S_{k}$ over all types $k$ weighted by the proportion of type- $k$ patch individuals, $u_{k}$, and thus we obtain

$$
\begin{aligned}
S_{\mathrm{IF}} & =\sum_{k=1}^{N} u_{k} S_{k} \\
& =\sum_{k=1}^{N} u_{k} v_{k}\left(-\gamma_{k}\right)\left(1-R_{k}\right) \\
& =\sum_{k=1}^{N} \underbrace{\frac{n_{k} \pi_{k}}{\langle n\rangle}}_{=u_{k}} \underbrace{\frac{\langle n\rangle}{\left\langle\frac{n F}{d}\right\rangle} \frac{F_{k, \text { res }}^{0}}{d_{k}}}_{=v_{k}} \underbrace{\frac{F_{k, \mathrm{~S}}}{F_{k, \text { res }}^{0}}}_{=-\gamma_{k}} \underbrace{\frac{d_{k}\left(2-d_{k}\right) n_{k}}{n_{k}-\left(n_{k}-1\right)\left(1-d_{k}\right)^{2}}}_{=1-R_{k}}
\end{aligned}
$$

Comparing with our expression of $D_{1}\left(s_{\text {res }}\right)$, we have

$$
\frac{\left\langle\frac{n F}{d}\right\rangle}{\langle n F\rangle} S_{\mathrm{IF}}=D_{1}\left(s_{\mathrm{res}}\right),
$$

which is consistent with eq.(F.24) of Parvinen et al. (2017) that reads

$$
\frac{S_{\mathrm{IF}}}{d}=D_{1}\left(s_{\mathrm{res}}\right)
$$

for a homogeneous case (this was first shown by Ajar (2003) in its eq.(8)). In particular, $S_{\mathrm{IF}}$ and $D_{1}\left(s_{\text {res }}\right)$ are sign-equivalent (Lehmann et al., 2016; Mullon et al., 2016).

\section{References}

Ajar, É. (2003). Analysis of disruptive selection in subdivided populations. $B M C$ Evolutionary Biology 3:22, 1-12.

Allen, B., M. A. Nowak, and U. Dieckmann (2013). Adaptive dynamics with interaction structure. Am. Nat. 181, E139-E163.

Boots, M., P. J. Hudson, and A. Sasaki (2004). Large shifts in pathogen virulence relate to host population structure. Science 303, 842-844.

Day, T. (2000). Competition and the effect of spatial resource heterogeneity on evolutionary diversification. Am. Nat. 155, 790-803.

Day, T. (2001). Population structure inhibits evolutionary diversification under competition for resources. Genetica 112-113, 71-86. 
Débarre, F. and S. Gandon (2011). Evolution in heterogeneous environments: between soft and hard selection. Am. Nat. 17\%, E84-E97.

Débarre, F., C. Hauert, and M. Doebeli (2014). Social evolution in structured populations. Nat. Comm. 5, 3409.

Dieckmann, U. and M. Doebeli (1999). On the origin of species by sympatric speciation. Nature 400, 354-357.

Dieckmann, U. and R. Law (1996). The dynamical theory of coevolution: A derivation from stochastic ecological processes. J. Math. Biol. 34, 579-612.

Doebeli, M. (2011). Adaptive Diversification. Princeton University Press, Princeton, NJ.

Doebeli, M. and U. Dieckmann (2003). Speciation along environmental gradients. Nature 421, 259-264.

Fletcher, J. A. and M. Doebeli (2009). A simple and general explanation for the evolution of altruism. Proc. R. Soc. London B 276, 13-19.

Frank, S. A. (1998). Foundations of Social Evolution. Princeton University Press, Princeton, NJ.

Gandon, S. and Y. Michalakis (1999). Evolutionarily stable dispersal rate in a metapopulation with extinctions and kin competition. J. Theor. Biol. 199, 275290.

Gandon, S. and F. Rousset (1999). Evolution of stepping-stone dispersal rates. Proc. R. Soc. London B 266, 2507-2513.

Geritz, S. A. H., É. Kisdi, G. Meszéna, and J. A. J. Metz (1998). Evolutionarily singular strategies and the adaptive growth and branching of the evolutionary tree. Evol. Ecol. 12, 35-57.

Geritz, S. A. H., J. A. J. Metz, É. Kisdi, and G. Meszéna (1997). Dynamics of adaptation and evolutionary branching. Phys. Rev. Lett. 78, 2024-2027.

Geritz, S. A. H., E. van der Meijden, and J. A. J. Metz (1999). Evolutionary dynamics of seed size and seedling competitive ability. Theor. Popul. Biol. 55, $324-343$.

Gyllenberg, M. and J. A. J. Metz (2001). On fitness in structured metapopulations. J. Math. Biol. 43, 545-560.

Haller, B. C., R. Mazzucco, and U. Dieckmann (2013). Evolutionary branching in complex landscapes. Am. Nat. 182, E127-E141.

Hamilton, W. D. (1964). The genetical evolution of social behaviour i. J. Theor. Biol 7, 1-16. 
Hauert, C. and M. Doebeli (2004). Spatial structure often inhibits the evolution of cooperation in the snowdrift game. Nature 428, 643-646.

Heinz, S. K., R. Mazzucco, and U. Dieckmann (2009). Speciation and the evolution of dispersal along environmental gradients. Evol. Ecol. 23, 53-70.

Irwin, A. J. and P. D. Taylor (2001). Evolution of altruism in steppingstone populations with overlapping generations. Theor. Popul. Biol. 60, 315-325.

Le Galliard, J.-F., R. Ferriére, and U. Dieckmann (2003). The adaptive dynamics of altruism in spatially heterogeneous populations. Evolution 57, 1-17.

Lehmann, L., M. W. Feldman, and F. Rousset (2009). On the evolution of harming and recognition in finite panmictic and infinite structured populations. Evolution 63, 2896-2913.

Lehmann, L. and L. Keller (2006). Synergy, partner choice and frequency dependence: their integration into inclusive fitness theory and their interpretation in terms of direct and indirect fitness effects. J. Evol. Biol 19, 1426-1436.

Lehmann, L., C. Mullon, E. Akçay, and J. Van Cleve (2016). Invasion fitness, inclusive fitness, and reproductive numbers in heterogeneous populations. Evolution 70, 1689-1702.

Lehmann, L. and F. Rousset (2010). How life history and demography promote or inhibit the evolution of helping behaviours. Phil. Trans. R. Soc. B 365, 2599-2617.

Lion, S. and M. van Baalen (2007). From infanticide to parental care: why spatial structure can help adults be good parents. Am. Nat. 170, E26-E46.

Mather, K. (1955). Polymorphism as an outcome of disruptive selection. Evolution 9, 52-61.

Maynard Smith, J. (1966). Sympatric speciation. Am. Nat. 100, 637-650.

Meszéna, G., I. Czibula, and S. A. H. Geritz (1997). Adaptive dynamics in a 2-patch environment: a toy model for allopatric and parapatric speciation. $J$. Biol. Syst. 5, 265-284.

Metz, J. A. J., S. A. H. Geritz, G. Meszéna, F. J. A. Jacobs, and J. S. van Heerwaarden (1996). Adaptive dynamics, a geometrical study of the consequenses of nearly faithful reproduction. In S. J. van Strien and S. M. Verduyn Lunel (Eds.), Stochastic and Spatial Structures of Dynamical Systems, pp. 183-231. North-Holland, Amsterdam.

Metz, J. A. J. and M. Gyllenberg (2001). How should we define fitness in structured metapopulation models? Including an application to the calculation of ES dispersal strategies. Proc. R. Soc. London B 268, 499-508. 
Metz, J. A. J. and O. Leimar (2011). A simple fitness proxy for structured populations with continuous traits, with case studies on the evolution of haplo-diploids and genetic dimorphisms. J. Biol. Dyn 5, 163-190.

Metz, J. A. J., R. M. Nisbet, and S. A. H. Geritz (1992). How should we define "fitness" for general ecological scenarios? Trends Ecol. Evol. 7, 198-202.

Mullon, C., L. Keller, and L. Lehmann (2016). Evolutionary stability of jointly evolving traits in subdivided populations. Am. Nat. 188, 175-195.

Mullon, C., L. Keller, and L. Lehmann (2018). Social polymorphism is favoured by the co-evolution of dispersal with social behaviour. Nature Ecology \& Evolution 2, 132-140.

Nakamaru, M., H. Matsuda, and Y. Iwasa (1997). The evolution of cooperation in a lattice-structured population. J. Theor. Biol. 184, 65-81.

Nowak, M. A. (2006). Evolutionary Dynamics. Harvard University Press, Cambridge, MA.

Nowak, M. A. and R. M. May (1992). Evolutionary games and spatial chaos. Nature 359, 826-829.

Nurmi, T. and K. Parvinen (2008). On the evolution of specialization with a mechanistic underpinning in structured metapopulations. Theor. Popul. Biol. 73, $222-243$.

Ohtsuki, H., C. Hauert, E. Lieberman, and M. A. Nowak (2006). A simple rule for the evolution of cooperation on graphs and social networks. Nature 441, $502-505$.

Ohtsuki, H. and M. A. Nowak (2006). The replicator equation on graphs. J. Theor. Biol. 243, 86-97.

Parvinen, K. (2002). Evolutionary branching of dispersal strategies in structured metapopulations. J. Math. Biol. 45, 106-124.

Parvinen, K., H. Ohtsuki, and J. Y. Wakano (2017). The effect of fecundity derivatives on the condition of evolutionary branching in spatial models. $J$. Theor. Biol 416, 129-143.

Payne, J. L., R. Mazzucco, and U. Dieckmann (2011). The evolution of conditional dispersal and reproductive isolation along environmental gradients. J. Theor. Biol. 273, 147-155.

Rodrigues, A. M. M. and A. Gardner (2012). Evolution of helping and harming in heterogeneous populations. Evolution 66-7, 2065-2079.

Rousset, F. (2004). Genetic structure and selection in subdivided populations. Princeton University Press, Princeton. 
Rousset, F. and O. Ronce (2004). Inclusive fitness for traits affecting metapopulation demography. Theor. Popul. Biol. 65, 127-141.

Sasaki, A. and G. de Jong (1999). Density dependence and upredictable selection in a heterogeneous environment: compromise and polymorphism in the ESS reaction norm. Evolution 53, 1329-1342.

Sigmund, K. (2010). The Calculus of Selfishness. Princeton University Press.

Szabó, G. and G. Fáth. (2007). Evolutionary games on graphs. Phys. Rep. 446, 97-216.

Tarnita, C. E., T. Antal, H. Ohtsuki, and M. A. Nowak (2009). Evolutionary dynamics in set structured populations. Proc. Natl. Acad. Sci. USA 106, 86018604 .

Taylor, P. D. (1990). Allele-frequency change in a class-structured population. Am. Nat. 135, 95-106.

Taylor, P. D. (1992a). Altruism in viscous populations - an inclusive fitness model. Evol. Ecol. 6, 352-356.

Taylor, P. D. (1992b). Inclusive fitness in a homogeneous environment. Proc. $R$. Soc. London B 249, 299-302.

Taylor, P. D., T. Day, and G. Wild (2007). Evolution of cooperation in a finite homogeneous graph. Nature 447, 469-472.

Taylor, P. D. and S. A. Frank (1996). How to make a kin selection model. J. Theor. Biol. 180, 27-37.

Taylor, P. D. and A. J. Irwin (2000). Overlapping generations can promote altruistic behavior. Evolution 54, 1135-1141.

van Baalen, M. and D. A. Rand (1998). The unit of selection in viscous populations and the evolution of altruism. J. Theor. Biol. 193, 631-648.

Wakano, J. Y. and L. Lehmann (2014). Evolutionary branching in deme-structured populations. J. Theor. Biol 351, 83-95.

Wild, G., A. Gardner, and S. A. West (2009). Adaptation and the evolution of parasite virulence in a connected world. Nature 459, 983-986.

Wilson, D. S., G. B. Pollock, and L. A. Dugatkin (1992). Can altruism evolve in a purely viscous population? Evol. Ecol. 6, 331-341.

Wright, S. (1931). Evolution in mendelian populations. Genetics 16, 97-159. 


\section{Electronic supplementary material: General- ization of the homogeneous theorems into the heterogeneous case}

This Appendix is similar to some appendices of Parvinen et al. (2017), and only given for completeness.

\section{C.1 Proof of the first-order results (Theorem 1)}

In this part of the Appendix, our aim is to provide a proof for Theorem 1, which gives an explicit expression for the selection gradient in terms of derivatives of the fecundity functions. This proof consists of two parts. As explained in Section 3, the vector $\omega_{k}$ needed in the calculation of metapopulation fitness is obtained by solving a system of linear equations. Therefore, we first use the implicit function theorem to obtain an explicit expression for the first derivative of metapopulation fitness. Second, by taking advantage of symmetry properties of the fecundity function, we obtain the equation presented in Theorem 1. Throughout the appendix we will use the notations

$$
L_{1}=\left\{1,2,3, \ldots, n_{k}\right\}^{T}, \text { and in more general, } \quad L_{j}=\left\{1^{j}, 2^{j}, 3^{j}, \ldots, n_{k}^{j}\right\}^{T} .
$$

The dimension of these vectors depends on the patch type through $n_{k}$, but for notational simplicity we simply write $L_{j}$ below, because the correct dimension is obvious from the context.

Equations involving vectors $L_{j}$, the vector $\omega_{k}$, and the matrix $\mathbf{T}_{k}$ needed in the proof are derived in C.3.

Since $R_{\mathrm{m}}=\sum_{k=1}^{N} \pi_{k} E_{k}^{T} \omega_{k}$, the metapopulation fitness gradient is

$$
D_{\mathrm{m}}\left(s_{\mathrm{res}}\right)=\left.\frac{\partial}{\partial s_{\mathrm{mut}}} R_{\mathrm{m}}\right|_{s_{\mathrm{mut}}=s_{\mathrm{res}}}=\sum_{k=1}^{N} \pi_{k}\left(E_{k}^{T} \omega_{k}^{\prime}+E_{k}^{\prime T} \omega_{k}\right) .
$$

Vectors $E_{k}$ and $\omega_{k}$ have an intuitive meaning. The elements $E_{k, i}$ describe the expected number of successful emigrants from a patch of type $k$ with $i$ mutants, and $\omega_{k, i}$ is the average time that a mutant colony in a patch of type $k$ spends in state with $i$ mutants (the sojourn time). The metapopulation fitness (reproduction number) $R_{\mathrm{m}}$ is the average number of successful emigrants of a mutant colony, where averaging is taken also over different patch types. The first component inside the parenthesis of (C.2), $\left(E_{k}^{T} \omega_{k}^{\prime}\right)$, describes how a (first-order) change in the sojourn time affects the total number of emigrants of the colony, provided that the emigrant production in each patch of type $k$ remains fixed. The second term $\left(E_{k}^{\prime T} \omega_{k}\right)$ describes the effect of changed emigrant production (first-order) in each patch of type $k$, provided that the sojourn times remain fixed. These two first-order components together, averaged over patch types, form the fitness gradient. 
Proposition 4. The metapopulation fitness gradient can be written as

$$
D_{\mathrm{m}}\left(s_{\mathrm{res}}\right)=\sum_{k=1}^{N} \pi_{k}\left[V_{k}\left(\frac{\partial}{\partial s_{\mathrm{mut}}} L_{1}^{T} \mathbf{T}_{k}\right) \omega_{k}+E_{k}^{\prime T} \omega_{k}\right]
$$

Proof. According to equation (2.15), the sojourn times $\omega_{k}$ are implicitly defined by $\left(\mathbf{I}-\mathbf{T}_{k}\right) \omega_{k}=\alpha_{k, 0}$. From the implicit function theorem we have

$$
\begin{aligned}
\omega_{k}^{\prime} & =\left.\frac{\partial}{\partial s_{\mathrm{mut}}} \omega_{k}\right|_{s_{\mathrm{mut}}=s_{\mathrm{res}}}=\left(\mathbf{I}-\mathbf{T}_{k}\right)^{-1}\left(-\left(\frac{\partial}{\partial s_{\mathrm{mut}}}\left(\mathbf{I}-\mathbf{T}_{k}\right)\right) \omega_{k}\right) \\
& =\left(\mathbf{I}-\mathbf{T}_{k}\right)^{-1}\left(\frac{\partial}{\partial s_{\mathrm{mut}}} \mathbf{T}_{k}\right) \omega_{k}
\end{aligned}
$$

According to (C.24) $L_{1}^{T}\left(\mathbf{I}-\mathbf{T}_{k}\right)=d_{k} L_{1}^{T}$ for $s_{\text {mut }}=s_{\text {res }}$. Based on (2.13) we then have

$$
E_{k}^{T}=\frac{p m n_{k} F_{k, \text { res }}^{0}}{I_{\text {res }}} d_{k} L_{1}^{T}=\frac{n_{k} F_{k, \text { res }}^{0}}{\langle n F\rangle} d_{k} L_{1}^{T}=V_{k} d_{k} L_{1}^{T} .
$$

Therefore,

$$
E_{k}^{T}\left(\mathbf{I}-\mathbf{T}_{k}\right)^{-1}=V_{k} L_{1}^{T}
$$

From (C.4) and (C.6) it follows that

$$
E_{k}^{T} \omega_{k}^{\prime}=V_{k} L_{1}^{T}\left(\frac{\partial}{\partial s_{\mathrm{mut}}} \mathbf{T}_{k}\right) \omega_{k}=V_{k}\left(\frac{\partial}{\partial s_{\mathrm{mut}}} L_{1}^{T} \mathbf{T}_{k}\right) \omega_{k},
$$

and thus (C.2) becomes (C.3).

Next, we want to write (C.3) in a more accessible form. For this purpose, by differentiating (2.3) and (2.4) we obtain

$$
\begin{aligned}
F_{k, \text { res }}^{\prime}(i) & =\left.\frac{\partial}{\partial s_{\mathrm{mut}}} F_{k, \text { res }}^{i}\left(s_{\mathrm{res}}, s_{\mathrm{mut}}\right)\right|_{s_{\mathrm{mut}}=s_{\mathrm{res}}}=i F_{k, \mathrm{D}} \\
F_{k, \text { mut }}^{\prime}(i) & =\left.\frac{\partial}{\partial s_{\mathrm{mut}}} F_{k, \text { mut }}^{i}\left(s_{\mathrm{res}}, s_{\mathrm{mut}}\right)\right|_{s_{\mathrm{mut}}=s_{\mathrm{res}}}=F_{k, \mathrm{~S}}+(i-1) F_{k, \mathrm{D}} .
\end{aligned}
$$

Let us now investigate the first term of (C.3). According to (C.23) we have $\left(L_{1}^{T} \mathbf{T}_{k}\right)_{i}=n_{k} p_{k, i}$. By differentiating $p_{k, i}$ (Equation 2.10) we obtain

$$
\begin{aligned}
&\left.\frac{\partial}{\partial s_{\mathrm{mut}}} n_{k} p_{k, i}\right|_{s_{\mathrm{mut}}=s_{\mathrm{res}}}=(1-m) i \frac{\left.\left[(1-m) n_{k} F_{k, \mathrm{res}}^{0}+I_{\mathrm{res}}\right)-(1-m) i F_{k, \text { res }}^{0}\right] F_{k, \mathrm{mut}}^{\prime}(i)-(1-m)\left(n_{k}-i\right) F_{k, \mathrm{res}}^{0} F_{k, \mathrm{res}}^{\prime}(i)}{\left((1-m) n_{k} F_{k, \mathrm{res}}^{0}+I_{\mathrm{res}}\right)^{2}} \\
&= \\
& \frac{1}{F_{k, \text { res }}^{0}}\left[i K_{1}+i^{2} K_{2}\right],
\end{aligned}
$$

where the second equality follows from (C.8) and (3.1). The coefficients $K_{i}$ are

$$
\begin{aligned}
& K_{1}=\left(1-d_{k}\right)\left(F_{k, S}-F_{k, D}\right) \\
& K_{2}=\frac{1-d_{k}}{n_{k}}\left[d_{k} n_{k} F_{k, D}-\left(1-d_{k}\right)\left(F_{k, S}-F_{k, D}\right)\right] .
\end{aligned}
$$


Therefore

$$
\frac{\partial}{\partial s_{\mathrm{mut}}} L_{1}^{T} \mathbf{T}_{k}=\frac{1}{F_{k, \mathrm{res}}^{0}}\left[K_{1} L_{1}+K_{2} L_{2}\right] .
$$

Then consider the second term of (C.3). By differentiating (2.13) we obtain

$$
E_{k, i}^{\prime}=\left.\frac{\partial}{\partial s_{\mathrm{mut}}} E_{k, i}\right|_{s_{\mathrm{mut}}=s_{\mathrm{res}}}=\frac{p m n_{k} d_{k}}{I_{\mathrm{res}}} i F_{k, \text { mut }}^{\prime}(i)=V_{k} \frac{d_{k}}{F_{k, \text { res }}^{0}} i F_{k, \text { mut }}^{\prime}(i)
$$

By using (C.8) we obtain

$$
E_{k}^{\prime}=V_{k} \frac{d_{k}}{F_{k, \text { res }}^{0}}\left[\left(F_{k, S}-F_{k, D}\right) L_{1}+F_{k, D} L_{2}\right] .
$$

By combining (C.3) with (C.11) and (C.13), and then applying expressions (C.28) for $L_{1}^{T} \omega_{k}$ and (C.30) for $L_{2}^{T} \omega_{k}$, we obtain (3.5).

\section{C.2 Proof of second-order results (Theorem 3)}

In this part of the appendix, we prove Theorem 3, which gives an explicit expression for the second derivative of the metapopulation fitness (with respect to the strategy of the mutant) in terms of derivatives of the fecundity function. Analogously to C.1, we first use the implicit function theorem, and then use symmetry properties of the fecundity function.

Differentiating $R_{\mathrm{m}}$ (Equation 2.16) two times we obtain

$$
\left.\frac{\partial^{2}}{\partial s_{\mathrm{mut}}^{2}} R_{\mathrm{m}}\right|_{s_{\mathrm{mut}}=s_{\mathrm{res}}}=\sum_{k=1}^{N} \pi_{k}\left[E_{k}^{T} \omega_{k}^{\prime \prime}+2 E_{k}^{\prime T} \omega_{k}^{\prime}+E_{k}^{\prime \prime T} \omega_{k}\right]
$$

where $E_{k}^{\prime \prime}=\left.\frac{\partial^{2}}{\partial s_{\text {mut }}^{2}} E_{k}\right|_{s_{\text {mut }}=s_{\mathrm{res}}}$. The second-order effects of a mutation on metapopulation fitness thus contain second-order effects on sojourn time $\omega_{k}$, provided that emigrant production $E_{k}$ remains fixed (first term), and second-order effects on emigrant production, provided that the sojourn time remains fixed (third term), and finally first-order effects on both (second term).

Proposition 5. The second derivative (C.14) can be written as

$$
\left.\frac{\partial^{2}}{\partial s_{\mathrm{mut}}^{2}} R_{\mathrm{m}}\right|_{s_{\mathrm{mut}}=s_{\mathrm{res}}}=\sum_{k=1}^{N} \pi_{k}\left[V_{k}\left(\frac{\partial^{2}}{\partial s_{\mathrm{mut}}^{2}} L_{1}^{T} \mathbf{T}_{k}\right) \omega_{k}+2\left[V_{k}\left(\frac{\partial}{\partial s_{\mathrm{mut}}} L_{1}^{T} \mathbf{T}_{k}\right)+E_{k}^{\prime T}\right] \omega_{k}^{\prime}+E_{k}^{\prime \prime T} \omega_{k}\right]
$$

Proof. Consider the terms of (C.14). We can use the implicit function theorem to obtain

$$
\begin{aligned}
\omega_{k}^{\prime \prime} & =\left.\frac{\partial^{2}}{\partial s_{\text {mut }}^{2}} \omega_{k}\right|_{s_{\text {mut }}=s_{\text {res }}}=\left(\mathbf{I}-\mathbf{T}_{k, \text { res }}\right)^{-1}\left[-\left(\left(\frac{\partial^{2}}{\partial s_{\text {mut }}^{2}}\left(\mathbf{I}-\mathbf{T}_{k}\right)\right) \omega_{k}+2\left(\frac{\partial}{\partial s_{\text {mut }}}\left(\mathbf{I}-\mathbf{T}_{k}\right)\right) \omega_{k}^{\prime}\right)\right] \\
& =\left(\mathbf{I}-\mathbf{T}_{k}\right)^{-1}\left(\left(\frac{\partial^{2}}{\partial s_{\text {mut }}^{2}} \mathbf{T}_{k}\right) \omega_{k}+2\left(\frac{\partial}{\partial s_{\text {mut }}} \mathbf{T}_{k}\right) \omega_{k}^{\prime}\right)
\end{aligned}
$$


Based on (C.16) and (C.6) we have for $s_{\text {mut }}=s_{\text {res }}$

$$
E_{k}^{T} \omega_{k}^{\prime \prime}=V_{k} L_{1}^{T}\left(\left(\frac{\partial^{2}}{\partial s_{\mathrm{mut}}^{2}} \mathbf{T}_{k}\right) \omega_{k}+2\left(\frac{\partial}{\partial s_{\mathrm{mut}}} \mathbf{T}_{k}\right) \omega_{k}^{\prime}\right)
$$

We investigate the three terms of the expression (C.15) for the second derivative in turns. First look at the component $\frac{\partial^{2}}{\partial s_{\text {mut }}^{2}}\left(L_{1}^{T} \mathbf{T}_{k}\right)$. According to (C.23) we have $\left(L_{1}^{T} \mathbf{T}_{k}\right)_{i}=n_{k} p_{k, i}$. By differentiating $p_{k, i}$ (Equation 2.10) and using

$$
\begin{aligned}
\left.\frac{\partial^{2}}{\partial s_{\mathrm{mut}}^{2}} F_{\mathrm{res}}^{i}\left(s_{\mathrm{res}}, s_{\mathrm{mut}}\right)\right|_{s_{\mathrm{mut}}=s_{\mathrm{res}}} & =i F_{k, \mathrm{DD}}+i(i-1) F_{k, \mathrm{DD}^{\prime}} \\
\left.\frac{\partial^{2}}{\partial s_{\mathrm{mut}}^{2}} F_{\mathrm{mut}}^{i}\left(s_{\mathrm{res}}, s_{\mathrm{mut}}\right)\right|_{s_{\mathrm{mut}}=s_{\mathrm{res}}} & =F_{k, \mathrm{SS}}+(i-1) F_{k, \mathrm{DD}}+2(i-1) F_{k, \mathrm{SD}} \\
& +(i-1)(i-2) F_{k, \mathrm{DD}^{\prime}} .
\end{aligned}
$$

we obtain

$$
\left.\frac{\partial^{2}}{\partial s_{\mathrm{mut}}^{2}} n_{k} p_{k, i}\right|_{s_{\mathrm{mut}}=s_{\mathrm{res}}}=A_{1} i+A_{2} i^{2}+A_{3} i^{3}
$$

where

$$
A_{1}=\frac{\left(1-d_{k}\right)\left(-F_{k, \mathrm{DD}}+2 F_{k, \mathrm{DD}}-2 F_{k, \mathrm{SD}}+F_{k, \mathrm{SS}}\right)}{F_{k, \mathrm{res}}^{0}} .
$$

Also the expressions for $A_{2}$ and $A_{3}$ depend on $d_{k}$ and the derivatives of the fecundity function, but they are quite lengthy. For details, see the electronic supplement of Parvinen et al. (2017). We obtain $\left(\frac{\partial^{2}}{\partial s_{\text {mut }}^{2}}\left(L_{1}^{T} \mathbf{T}_{k}\right)\right) \omega_{k}=\left(A_{1} L_{1}^{T}+A_{2} L_{2}^{T}+\right.$ $\left.A_{3} L_{3}^{T}\right) \omega_{k}$, and by using (C.28), (C.30) and (C.31) we get the first part ready. It is not shown separately, since we only need the sum in (C.15).

Concerning the second term, $\frac{\partial}{\partial s_{\mathrm{mut}}}\left(L_{1}^{T} \mathbf{T}_{k}\right)$ is given by (C.11) and $E_{k}^{\prime T}$ is given by (C.13). We need to calculate their product with $\omega_{k}^{\prime}$, obtained from (C.4). For this purpose we first need expressions (C.32) and (C.33) for $L_{j}\left(\mathbf{I}-\mathbf{T}_{k}\right)^{-1}$, thereafter (C.11) and (C.34) for $\frac{\partial}{\partial s_{\mathrm{mut}}}\left(L_{j} \mathbf{T}_{k}\right)$, and finally (C.28), (C.30) and (C.31) for $L_{i}^{T} \omega_{k}$ to obtain an explicit expression (not shown separately).

The third term is obtained by differentiating $E_{k}(2.13)$ :

$$
E_{k, i}^{\prime \prime}=\left.\frac{\partial^{2}}{\partial s_{\mathrm{mut}}^{2}} E_{k, i}\right|_{s_{\mathrm{mut}}=s_{\mathrm{res}}}=V_{k} \frac{d_{k}}{F_{k, \mathrm{res}}^{0}} i F_{k, \mathrm{mut}}^{\prime \prime}(i) .
$$

Therefore,

$$
E_{k}^{\prime \prime} \omega_{k}=V_{k} \frac{d_{k}}{F_{k, \mathrm{res}}^{0}}\left(C_{1} L_{1}+C_{2} L_{2}+C_{3} L_{3}\right) \omega_{k}
$$


where $C_{1}=-F_{k, \mathrm{DD}}+2 F_{k, \mathrm{DD}^{\prime}}-2 F_{k, \mathrm{SD}}+F_{k, \mathrm{SS}}, C_{2}=F_{k, \mathrm{DD}}-3 F_{k, \mathrm{DD}^{\prime}}+2 F_{k, \mathrm{SD}}$ and $C_{3}=F_{k, \mathrm{DD}^{\prime}}$. By applying (C.28), (C.30) and (C.31) for $L_{i}^{T} \omega_{k}$ we obtain an explicit expression for $E^{\prime \prime} \omega_{k}$ (not shown separately).

The final result (Equation 3.10 of Theorem 3) is obtained by adding together the three expressions mentioned above.

\section{C.3 Vectors $L_{1}, L_{2}$ and $L_{3}$}

The proofs of the results in this subsection are all straightforward generalizations of those presented by Parvinen et al. (2017), except for the proof of (C.28). Note that most of the equations in this subsection are such that they are valid only for $s_{\text {mut }}=s_{\text {res }}$.

\section{C.3.1 The vectors $L_{i}^{T} \mathbf{T}_{k}$}

According to the definition of (2.11), we have

$$
\left(L_{1}^{T} \mathbf{T}_{k}\right)_{i}=\sum_{j=1}^{n_{k}} j t_{j i}=\sum_{j=1}^{n_{k}} j\left(\begin{array}{c}
n_{k} \\
j
\end{array}\right)\left(p_{k, i}\right)^{j}\left(1-p_{k, i}\right)^{n_{k}-j}=\mathrm{E}\left(\mathbf{X}_{i}\right)=n_{k} p_{k, i}
$$

where $\mathbf{X}_{i}$ is a binomially distributed random variable with parameters $n_{k}$ and $p_{k, i}$. According to $(2.10)$ and $(3.1), p_{k, i}=\frac{(1-m) i F_{k, \text { res }}^{0}}{(1-m) n_{k} F_{k, \text { res }}^{0}+I_{\text {res }}}=\frac{\left(1-d_{k}\right) i}{n_{k}}$ for the resident, and thus

$$
L_{1}^{T} \mathbf{T}_{k}=\left(1-d_{k}\right) L_{1}^{T}
$$

Analogously, we have

$$
\begin{aligned}
\left(L_{2}^{T} \mathbf{T}_{k}\right)_{i} & =\sum_{j=1}^{n_{k}} j^{2} t_{j i}=\sum_{j=1}^{n_{k}} j^{2}\left(\begin{array}{c}
n_{k} \\
j
\end{array}\right)\left(p_{k, i}\right)^{j}\left(1-p_{k, i}\right)^{n_{k}-j}=\mathrm{E}\left(\mathbf{X}_{i}^{2}\right) \\
& =n_{k} p_{k, i}+n_{k}\left(n_{k}-1\right) p_{k, i}^{2} .
\end{aligned}
$$

Again, by using (2.10) and (3.1) we obtain

$$
\left(L_{2}^{T} \mathbf{T}_{k}\right)_{i}=\left(1-d_{k}\right) i+\frac{\left(n_{k}-1\right)\left(1-d_{k}\right)^{2}}{n_{k}} i^{2} .
$$

In a similar way, we have

$$
\begin{aligned}
\left(L_{3}^{T} \mathbf{T}_{k}\right)_{i} & =\sum_{j=1}^{n_{k}} j^{3} t_{j i}=\sum_{j=1}^{n_{k}} j^{3}\left(\begin{array}{c}
n_{k} \\
j
\end{array}\right)\left(p_{k, i}\right)^{j}\left(1-p_{k, i}\right)^{n_{k}-j}=\mathrm{E}\left(\mathbf{X}_{i}^{3}\right) \\
& =n_{k} p_{k, i}+3 n_{k}\left(n_{k}-1\right) p_{k, i}^{2}+n_{k}\left(n_{k}-1\right)\left(n_{k}-2\right) p_{k, i}^{3} \\
& =\left(1-d_{k}\right) i+3 \frac{\left(n_{k}-1\right)\left(1-d_{k}\right)^{2}}{n_{k}} i^{2}+\frac{\left(n_{k}-1\right)\left(n_{k}-2\right)\left(1-d_{k}\right)^{3}}{n_{k}^{2}} i^{3} .
\end{aligned}
$$




\section{C.3.2 The scalars $L_{i}^{T} \omega_{k}$}

According to (C.24) $L_{1}^{T} \mathbf{T}_{k}=\left(1-d_{k}\right) L_{1}^{T}$. By applying it several times we get $L_{1}^{T}\left(\mathbf{T}_{k}\right)^{t}=\left(1-d_{k}\right)^{t} L_{1}^{T}$, and therefore for each $k$ we have

$$
L_{1}^{T} \omega_{k}=L_{1}^{T} \sum_{t=0}^{\infty}\left(\mathbf{T}_{k}\right)^{t} \alpha_{k, 0}=L_{1}^{T} \sum_{t=0}^{\infty}\left(1-d_{k}\right)^{t} \alpha_{k, 0}=\frac{1}{d_{k}} L_{1}^{T} \alpha_{k, 0}=\frac{1}{d_{k}}
$$

Note that the homogeneous version of (C.28) was proved by Parvinen et al. (2017) using the equation $1=R_{\mathrm{m}}=E^{T} \omega=d L_{1}^{T} \omega$, which does not imply (C.28) in the heterogeneous case. Therefore, we proved it in a different way above.

According to (2.14) we have $\mathbf{T}_{k} \omega_{k}=\omega_{k}-\alpha_{k, 0}$, so that

$$
L_{i}^{T} \mathbf{T}_{k} \omega_{k}=L_{i}^{T}\left(\omega_{k}-\alpha_{k, 0}\right)=L_{i}^{T} \omega_{k}-1 .
$$

By using (C.26) and (C.28), the equation (C.29) with $i=2$ gets a form from which $L_{2}^{T} \omega_{k}$ can be solved:

$$
L_{2}^{T} \omega_{k}=\frac{n_{k}}{d_{k}\left(1+\left(n_{k}-1\right) d_{k}\left(2-d_{k}\right)\right)} .
$$

In a similar way, by using (C.27) and (C.29) with $i=3$ together with results above, we can solve

$$
L_{3}^{T} \omega_{k}=\frac{\left(n_{k}+2\left(n_{k}-1\right)\left(1-d_{k}\right)^{2}\right) n_{k}^{2}}{d_{k}\left(1+\left(n_{k}-1\right) d_{k}\left(2-d_{k}\right)\right)\left(n_{k}^{2}-\left(n_{k}-1\right)\left(n_{k}-2\right)\left(1-d_{k}\right)^{3}\right)} .
$$

\section{C.3.3 Vectors $L_{i}^{T}\left(\mathbf{I}-\mathbf{T}_{k}\right)^{-1}$}

From (C.24) we get $L_{1}^{T}\left(\mathbf{I}-\mathbf{T}_{k}\right)=d_{k} L_{1}^{T}$ so that

$$
L_{1}^{T}\left(\mathbf{I}-\mathbf{T}_{k}\right)^{-1}=\frac{1}{d_{k}} L_{1}^{T} .
$$

Furthermore, from (C.26) we have $L_{2}^{T}\left(\mathbf{I}-\mathbf{T}_{k}\right)=\left(d_{k}-1\right) L_{1}^{T}+\left(1-\frac{\left(1-d_{k}\right)^{2}\left(n_{k}-1\right)}{n_{k}}\right) L_{2}^{T}$. By multiplying with $\left(\mathbf{I}-\mathbf{T}_{k}\right)^{-1}$ from the right we get an expression from which we can solve

$$
L_{2}^{T}\left(\mathbf{I}-\mathbf{T}_{k}\right)^{-1}=\frac{n_{k}}{n_{k}-\left(n_{k}-1\right)\left(1-d_{k}\right)^{2}}\left(\frac{1-d_{k}}{d_{k}} L_{1}^{T}+L_{2}^{T}\right)
$$

\section{C.3.4 Vectors $L_{i}^{T} \mathbf{T}_{k}^{\prime}$}

The expression for $L_{1}^{T} \mathbf{T}_{k}^{\prime}$ was already obtained in (C.11). By differentiating (C.25) and using (2.10) and (3.1) we obtain 


$$
\begin{aligned}
L_{2}^{T} \mathbf{T}_{k}^{\prime}= & \frac{1}{F_{k, \text { res }}^{0}}\left(\left(1-d_{k}\right)\left(F_{k, \mathrm{~S}}-F_{k, \mathrm{D}}\right) L_{1}^{T}\right. \\
& +\frac{\left(1-d_{k}\right)}{n_{k}}\left(\left(1-d_{k}\right)\left(2 n_{k}-3\right) F_{k, \mathrm{~S}}+\left(3\left(1-d_{k}\right)+n_{k}\left(3 d_{k}-2\right)\right) F_{k, \mathrm{D}}\right) L_{2}^{T} \\
& \left.+2 \frac{\left(1-d_{k}\right)^{2}\left(n_{k}-1\right)}{n_{k}^{2}}\left(\left(1+d_{k}\left(n_{k}-1\right)\right) F_{k, \mathrm{D}}-\left(1-d_{k}\right) F_{k, \mathrm{~S}}\right) L_{3}^{T}\right) .
\end{aligned}
$$

